\title{
Analysis of Quantized MRC-MRT Precoder For FDD Massive MIMO Two-Way AF Relaying
}

\author{
B. Dutta, R. Budhiraja, R. D. Koilpillai and L. Hanzo Fellow IEEE
}

\begin{abstract}
The maturing massive multiple-input multipleoutput (MIMO) literature has provided asymptotic limits for the rate and energy efficiency ( $\mathrm{EE}$ ) of maximal ratio combining /maximal ratio transmission (MRC-MRT) relaying on two-way relays (TWR) using the amplify-and-forward (AF) principle. Most of these studies consider time division duplexing, and a fixed number of users. To fill the gap in the literature, we analyze the MRC-MRT precoder performance of a $N$-antenna AF massive MIMO TWR, which operates in frequency division duplex mode to enable two-way communication between $2 M=\left\lfloor N^{\alpha}\right\rfloor$ singleantenna users, with $\alpha \in[0,1)$, divided equally in two groups of $M$ users. We assume that the relay has realistic imperfect uplink channel state information (CSI), and that quantized downlink CSI is fed back by the users relying on $B \geq 1$ bits peruser per relay antenna. We prove that for such a system with $\alpha \in[0,1)$, the MRC-MRT precoder asymptotically cancels the multi-user interference (MUI) when the supremum and infimum of large scale fading parameters is strictly non-zero and finite, respectively. Furthermore, its per-user pairwise error probability (PEP) converges to that of an equivalent AWGN channel as both $N$ and the number of users $2 M=\left\lfloor N^{\alpha}\right\rfloor$ tend to infinity, with a relay power scaling of $P_{r}=\frac{2 M E_{r}}{N}$ and $E_{r}$ being a constant. We also derive upper bounds for both the per-user rate and EE. We analytically show that the quantized MRC-MRT precoder requires as few as $B=2$ bits to yield a BER, EE, and per-user rate close to the respective unquantized counterparts. Finally, we show that the analysis developed herein to derive a bound on $\alpha$ for MUI cancellation is applicable both to Gaussian as well as to any arbitrary non-Gaussian complex channels.
\end{abstract}

Index Terms-Asymptotic analysis, limited feedback, multiuser systems, pairwise error probability.

\section{INTRODUCTION}

$\mathbf{R}$ ELAY-based co-operative communication provides reliable links for users who do not have a direct link to communicate among themselves [1], [2]. Conventional relaying, commonly known as one-way relaying [1], achieves this objective but requires four distinct time/frequency channel uses for bi-directional communication between two users. By contrast, two-way relaying (TWR) only requires two channel uses for bidirectional communication [3], [4], hence improves the system's spectral efficiency compared to one-way relaying. In the first channel use of TWR, also known as the multiple access (MAC) phase, the two users who want to exchange data, transmit their respective signals to the relay, which receives a sum of these two signals. In the second channel

B. Dutta and R. D. Koilpillai are with the Department of Electrical Engineering, IIT Madras, Chennai-600036 (email: biswajit.dutta@gmail.com, davidk@iitm.ac.in).

R. Budhiraja is with the Department of Electrical Engineering IIT Kanpur, Kanpur-208016 (email: rohitbr@iitk.ac.in).

L.Hanzo is with the School of Electronics and Computer Science, University of Southampton, Southampton SO17 1BJ, U.K (email:lh@ecs.soton.ac.uk) use of TWR, also known as broadcast (BC) phase, the relay amplifies and forwards the sum-signal back to both the users. Since both users know their own data, they can cancel their own contribution from the received sum signal to recover the desired data. Bi-directional communication between two users is thus completed in just two channel uses.

The TWR concept has also been extended to multi-user scenarios, where multiple users simultaneously communicate via a relay. The users now experience multi-user interference (MUI) along with self interference (SI) [5]-[9]. Various designs have been conceived for canceling the MUI by using a multiple-input multiple output (MIMO) relay and for optimizing diverse metrics, such as the sum-rate, and the rate of the user having the weakest signal-to-interference-plus-noise ratio (SINR) [5]-[9]. The authors of [5], [6] constructed precoders for the relay based on the zero-forcing and the linear minimum mean squared error criteria respectively, for canceling both the SI and the MUI experienced by the users. Gunduz et al. [7] characterized the sum-rate of the multi-user Gaussian TWR while Fang et al. [8] designed a relay precoder for maximizing the SINR of the weakest user. Yuan [9] considered a clustered data exchange model and constructed beamforming matrices both for the relay and for the users with the aid of signal alignment. Yuan also determined the degrees of freedom for the system model considered in [9].

A massive MIMO transmitter equipped with a large number of antennas is also capable of mitigating the MUI by using low-complexity precoding techniques at the transmitter such as maximum ratio transmission (MRT) [10], [11]. A massive MIMO system is potentially capable of improving the sumrate and the energy-efficiency (EE) of wireless systems [10], [11]. Recently, massive MIMO technology has also been incorporated in TWR by employing a large number of antennas at the relay [12]-[18]. Cui et al. [12] constructed a precoder for the relay based on MRT and maximal ratio combining (MRC). Dai and Dong [13] studied the impact of imperfect channel estimation on the sum rate of massive MIMO multiuser TWR systems. Both [12] and [13] analyze the sumrate and $\mathrm{EE}$ by fixing the number of users while increasing the number of relay antennas. Liu et al. [14] constructed a reduced-feedback-based hybrid precoder for TWR systems, and showed that if the number of relay antennas and RF chains satisfy a given constraint, the transmit power of both the users and relays can be scaled down upon increasing the number of relay antennas without affecting the sumrate. Kong et al. [15] derived closed-form expressions for the spectral efficiency of massive MIMO TWR networks, which can also be used for studying power-scaling laws. 
Jin et al. [16] showed that the ergodic user rates increase upon increasing the number of relay antennas, but decrease with the number of users. Naghsh et al. [17] maximized the sum-rate of MIMO-aided TWR amplify-and-forward (AF) networks using a novel minorization-maximization based iterative algorithm. Reference [18] recently optimized the energy efficiency of a two-way AF massive MIMO relay.

All the aforementioned massive MIMO TWR contributions assume time division duplexing (TDD), where the uplink channel of the MAC phase and the downlink channel of the BC phase are assumed to be reciprocal. Based on reciprocity, the relay of a TDD system can readily infer the downlink channel state information (CSI) from the uplink CSI. For frequency division duplex (FDD) systems, where the uplink (UL) and downlink (DL) channels are non-reciprocal, the users feed back the quantized DL CSI to the relay.

The existing massive MIMO TWR literature has not yet analyzed the impact of quantized feedback in FDD systems on the asymptotic performance of different precoder design metrics such as the MUI-cancellation capability, pairwise error probability (PEP), EE, and per-user rate. Furthermore, it is also crucial to analyze these metrics upon scaling the number of users with the number of relay antennas noting that [12], [13] fixed them. Against this backdrop, the main contributions of this paper are as follows:

1) We consider a massive MIMO FDD multi-user TWR system having $N$ relay antennas and $2 M$ single-antenna users divided into two groups of $M$ users. The performance of the relay's MRC-MRT precoder is analyzed for the scenario, when it is designed using the UL CSI estimated by the relay, and DL CSI, estimated, quantized and fed back by the users.

2) We analytically show that when each of the $2 M$ users feeds back $B \geq 1$ bits per relay antenna, with the total number of users scaling as $2 M=\left\lfloor N^{\alpha}\right\rfloor$ with $\alpha \in[0,1)$, the MRC-MRT precoder asymptotically cancels the MUI, provided that the power of the relay scales as $P_{r}=\frac{2 M E_{r}}{N}$, where $E_{r}$ is a constant. We derive the symbol-PEP, and almostsure upper-bounds on the per-user rate and the system's EE. We show that the symbol-PEP of the quantized MRC-MRT precoder converges almost surely to the PEP of an AWGN channel. The current analysis is important because we i) consider a FDD relay and design the MRC-MRT precoder for using quantized channel feedback; ii) scale the number of users, sub-linearly, with $N$; and iii) show that the MRCMRT precoder designed using these constraints still achieves AWGN-like bit error rate (BER) for $N \rightarrow \infty$. In [12], [13] the authors designed precoders for a TDD relay (consequently assumed the availability of unquantized CSI) and analyzed the performance by fixing the number of users as $N \rightarrow \infty$. The analysis of per-user rate and of the EE of the quantized MRCMRT precoder, where the number of users scale sub-linearly with $N$, is completely different from that in [12], [13]. We rely on the moment expansion methods of Tao [19] and on the limit theorems of [20] for our analysis.

3) We analytically show that the quantized MRC-MRT precoder requires $B=2$ bits to approach the BER, the per-user rate and the EE of its unquantized counterparts in [12], [13]. 4) The authors of [12], [13] analyze the asymptotic EE by assuming that all users have identical large scale fading parameters. We, in contrast, calculate the almost sure upper bounds on the per-user rate and on the EE by assuming different large scale fading parameters for all users, which makes analysis more realistic. We show that for ensuring uniform convergence of the EE upper bound derived, the supremum and infimum of large scale fading parameters should be finite and non-zero.

5) Although we compute the precoder's performance asymptotes for the Gaussian channels, we show that the analysis is also applicable for the zero-mean, complex non-Gaussian distribution with certain constraints on the probability density function (pdf) of the channel phases. This is unlike the work in [12], [13] which is valid only for Gaussian channels and that too for unquantized MRC-MRT precoder design.

The importance of this work is that the considered system model can be readily applied to control and telemetry applications like unmanned aerial vehicles (UAV's) [21], robotic cars [22], high-speed trains [23] which demand a very low BER, but not a very high data rate. These applications are typically scheduled on a time/frequency resource different from the remaining non-control applications. Further, these applications form a small fraction of the total users in the system. Scheduling such small number of applications in a massive MIMO system implies low user-antenna ratio, a scenario considered in this work.

With $2 M=N^{\alpha}$ constraint, we investigate 1) how should the relay antennas $N$ scale as the number of nodes i.e., UAV's, robotic cars or high-speed trains, increase, such that each node asymptotically achieves the reliable AWGN BER. 2) For a given $\alpha \in[0,1)$, the maximum $2 M$ number of nodes that can experience reliable AWGN BER for a given large $N$.

The rest of the paper is organized as follows. The system model is presented in Section II. The quantized MRC-MRT precoder design is introduced in Section III. For this precoder, we analytically characterize the asymptotic symbol PEP, peruser rate and the EE in Section IV. We then numerically evaluate these metrics in Section V.

Notation: We use boldface lower- and upper-case letters to denote vectors and matrices, respectively. The symbols $|\cdot|$, $(\cdot)^{*}$ and $\lfloor\cdot\rfloor$ denote the magnitude, complex conjugate of a complex number, and the floor of a real number, respectively. The symbol $\stackrel{a . s .}{\rightarrow}$ denotes the convergence of a random variable in the almost sure sense. A circularly-symmetric complex Gaussian random variable with a variance $N_{0}$ is denoted as $\mathcal{C N}\left(0, N_{0}\right)$. The notation $[\mathbf{A}]_{k l}$ represents the $(k, l)$ th entry of the matrix $\mathbf{A}$. For an $N \times N$ matrix $\mathbf{A}$, $\operatorname{Tr}(\mathbf{A})=\sum_{i=1}^{N}[\mathbf{A}]_{i i}$. The symbol $\mathbb{E}[\cdot]$ represents the expectation operator. The notation $f(x)=O[g(x)]$, means that there exist constants $k_{1}$ and $k_{2}$ such that $k_{1} g(x) \leq f(x) \leq k_{2} g(x)$. Further, blk-diag $\left\{\mathbf{P}_{1}, \cdots, \mathbf{P}_{M}\right\}$ means the block diagonal $\operatorname{matrix}\left[\begin{array}{cccc}\mathbf{P}_{1} & \mathbf{0} & \cdots & \mathbf{0} \\ \mathbf{0} & \mathbf{P}_{2} & \cdots & \mathbf{0} \\ \vdots & \vdots & \vdots & \vdots \\ \mathbf{0} & \cdots & \mathbf{0} & \mathbf{P}_{M}\end{array}\right]$ where $\mathbf{0}$ is the all zero matrix of appropriate dimensions. Next, the notation $\mathbf{D}^{1 / 2}$ implies a square matrix $\mathbf{B}$ such that $\mathbf{B}^{2}=\mathbf{D}$. 


\section{SySTEM MODEL}

We consider an AF single-cell massive MIMO TWR network as in [12], [13], where $M$ single-antenna user pairs communicate with each other via a relay equipped with $N$ antennas; the total number of users is given by $2 M=\left\lfloor N^{\alpha}\right\rfloor$. Without loss of generality, we assume that the $(2 k-1)$ st and $(2 k)$ th user communicate with each other via the relay. This is accomplished in two phases, each requiring a single channel use. In the MAC phase, each of the $2 M$ singleantenna users simultaneously transmits its respective data to the relay, which receives a signal vector $\mathbf{y}_{\mathbf{r}} \in \mathbb{C}^{N \times 1}$ given as $\mathbf{y}_{r}=\sum_{i=1}^{2 M} \sigma_{i} \mathbf{h}_{i} x_{i}+\mathbf{n}_{r}=\mathbf{H D}^{\frac{1}{2}} \mathbf{X}+\mathbf{n}_{r}$. Similar to [12], [13], the vector $\mathbf{h}_{i} \in \mathbb{C}^{N \times 1}$ denotes the UL channel spanning from the $i$ th user to the relay whose entries are assumed to be independent and identically distributed (iid) with a pdf $\mathcal{C N}(0,1)$. The scalar $\sigma_{i}$, similar to [12], [13], denotes the large scale fading coefficient of the $i$ th user. The scalar $x_{i}$ is the symbol transmitted by user $i$. The augmented matrix $\mathbf{H}=\left[\mathbf{h}_{1} \mathbf{h}_{2}, \cdots, \mathbf{h}_{2 M-1} \mathbf{h}_{2 M}\right] \in \mathbb{C}^{N \times 2 M}$ and the vector $\mathbf{x}=\left[x_{1} x_{2}, \cdots, x_{2 M-1} x_{2 M}\right]^{T}$ contain the uplink channels and independent transmit symbols of all users, respectively. The matrix $\mathbf{D}$ is a $2 M \times 2 M$ diagonal matrix with $[\mathbf{D}]_{i i}=\sigma_{i}^{2}$. The vector $\mathbf{n}_{r} \in \mathbb{C}^{N \times 1}$ denotes the relay receiver's noise with iid entries and pdf $\mathcal{C N}\left(0, N_{0}\right)$.

In the $\mathrm{BC}$ phase, the relay multiplies its received MACphase signal $\mathbf{y}_{r}$ by a precoder $\mathbf{F}$ as $\mathbf{x}_{r}=c \mathbf{F} \mathbf{y}_{r}$, and then forwards this signal to the $2 M$ users in the DL. The scalar $c$ ensures that the average relay transmit power constraint $P_{r}$ is met i.e.,

$$
c=\sqrt{\frac{P_{r}}{\mathbb{E}\left[\left\|\mathbf{F} \mathbf{y}_{r}\right\|^{2}\right]}} .
$$

The signal received by the $i^{\prime}$ th user is

$$
y_{i^{\prime}}=\sigma_{i^{\prime}} \mathbf{g}_{i^{\prime}} \mathbf{x}_{r}+n_{i^{\prime}}=\sigma_{i^{\prime}} \mathbf{g}_{i^{\prime}} \mathbf{F} \mathbf{y}_{r}+n_{i^{\prime}},
$$

where $\mathrm{g}_{i^{\prime}} \in \mathbb{C}^{N}$ denotes the row vector DL channel spanning from the relay to the $i^{\prime}$ th user, whose entries are iid $\mathcal{C N}(0,1)$. We note that $i^{\prime}=2 k$ and $i=2 k-1$ form a communicating pair. The augmented matrix $\mathbf{G}=$ $\left[\mathbf{g}_{1}^{T} \mathbf{g}_{2}^{T}, \cdots, \mathbf{g}_{2 M-1}^{T} \mathbf{g}_{2 M}^{T}\right]^{T} \in \mathbb{C}^{2 M \times N}$ contains the downlink channels of all the users. The scalar $n_{i^{\prime}}$ denotes the noise at the $i^{\prime}$ th user and has a pdf of $\mathcal{C N}\left(0, N_{0}\right)$.

Remark 1: We assume that the relay estimates the uplink channel $\mathbf{H}$ as $\widehat{\mathbf{H}}=\mathbf{H D}^{\frac{1}{2}}+\mathbf{E}_{\mathrm{u}}$. Here $\mathbf{E}_{\mathrm{u}}$ is the estimation error matrix, whose entries are iid with pdf of $\mathcal{C N}\left(0, \eta_{u}^{2}\right)$, and are independent of the entries of $\mathbf{H}$ [24], [25]. Let $\hat{\mathbf{h}}_{i}$ denote the $i$ th column of $\widehat{\mathbf{H}}$. Since we consider a FDD relay, the UL and DL channels are non-reciprocal. The DL channel $\mathbf{G}$ is available to the relay in its quantized form, fed back to it by the users.

We also assume that the $i^{\prime}$ th user estimates the DL channel vector $\sigma_{i^{\prime}} \mathbf{g}_{i^{\prime}}$. Based on its estimate of the channel gain vector $\sigma_{i^{\prime}} \mathbf{g}_{i^{\prime}}$, the $i^{\prime}$ th user computes $B$ bits per relay transmit antenna and feeds them back to the relay through an error-free lowrate feedback channel. The relay then constructs the precoder $\mathbf{F}$ based on the knowledge of $\widehat{\mathbf{H}}$ and the quantized knowledge of $\widehat{\mathbf{G}}=\mathbf{D}^{\frac{1}{2}} \mathbf{G}+\mathbf{E}_{\mathrm{d}}$. The matrix $\mathbf{E}_{\mathrm{d}}$ is the estimation error matrix with iid entries that are independent of the entries of $\mathbf{G}$ and have pdf of $\mathcal{C N}\left(0, \eta_{d}^{2}\right)$ [13]. We also use $\hat{\mathbf{g}}_{i^{\prime}}$ to denote the $i^{\prime}$ th row of the estimated DL channel matrix $\widehat{\mathbf{G}}$.

We now precisely state how the uplink channel is estimated at the relay, and the downlink channel is estimated and fed back to the relay.

Uplink channel estimation at the relay: We consider a blockfading channel with coherence time of $T_{c}$ samples. Each user sends a $T$-sample long $\left(2 M \leq T \ll T_{c}\right)$ complex orthogonal pilot row vector sequence $\mathbf{s}_{n} \in \mathbb{C}^{T}$ with power $P_{l}$ such that $\mathbf{s}_{n} \mathbf{s}_{n}^{H}=P_{l}$ and $\mathbf{s}_{n} \mathbf{s}_{n^{\prime}}^{H}=0$ for $n \neq n^{\prime}$ [11]. The received signal $\mathbf{Y} \in \mathbb{C}^{N \times T}$ over $\mathrm{T}$ samples is then given as

$$
\mathbf{Y}=\sigma_{n} \mathbf{h}_{n} \mathbf{s}_{n}+\sum_{k \neq n}^{2 M} \sigma_{k} \mathbf{h}_{k} \mathbf{s}_{k}+\mathbf{N}
$$

where $\mathbf{N} \in \mathbb{C}^{N \times T}$ is the relay noise with iid entries and pdf $\mathcal{C N}\left(0, N_{0}\right)$. The least squares estimate [24], [25] of $\mathbf{h}_{n}$ is then given as

$$
\hat{\mathbf{h}}_{n}=\frac{\mathbf{Y} \mathbf{s}_{n}^{H}}{P_{l}}=\sigma_{n} \mathbf{h}_{n}+\frac{\mathbf{N s}_{n}^{H}}{P_{l}} .
$$

Equation (3) shows that the error column vector $\mathbf{e}_{\text {un }}=\frac{\mathbf{N s}_{n}^{H}}{P_{l}} \in$ $\mathbb{C}^{N}$ is independent of $\mathbf{h}_{n}$, and its entries are iid with pdf $\mathcal{C N}\left(0, \frac{N_{0}}{P_{l}}\right)$. This implies that in the estimated uplink channel matrix $\widehat{\mathbf{H}}=\left[\begin{array}{lll}\hat{\mathbf{h}}_{1} & \cdots & \hat{\mathbf{h}}_{2 M}\end{array}\right]=\mathbf{H D}^{\frac{1}{2}}+\mathbf{E}_{u}$, the error matrix $\mathbf{E}_{u}$ has entries independent of the uplink channel matrix $\mathbf{H}=$ $\left[\begin{array}{lll}\mathbf{h}_{1} & \cdots & \mathbf{h}_{2 M}\end{array}\right]$, where $\mathbf{E}_{u}=\left[\begin{array}{lll}\mathbf{e}_{u 1} & \cdots & \mathbf{e}_{u 2 M}\end{array}\right]$ and $\mathbf{D}$ is a $2 M \times 2 M$ diagonal matrix with $[\mathbf{D}]_{i i}=\sigma_{i}^{2}$.

Downlink channel estimation at the users and its quantization: For the downlink channel of coherence time $T_{c}$ samples, the relay sends a complex orthogonal pilot sequence $\mathbf{S} \in \mathbb{C}^{N \times T}, 2 M \leq T \ll T_{c}$, with power $P_{l}$ such that $\mathbf{S S}^{H}=P_{l} \mathbf{I} \in \mathbb{C}^{N \times \bar{N}}$ [11]. The received symbol row vector $\mathbf{y}_{n} \in \mathbb{C}^{T}$ at user $n$ is

$$
\mathbf{y}_{n}=\sigma_{n} \mathbf{g}_{n} \mathbf{S}+\mathbf{n}_{n}
$$

where $\mathbf{n}_{n} \in \mathbb{C}^{T}$ is the thermal noise row vector at user $n$ with entries distributed iid $\mathcal{C N}\left(0, N_{0}\right)$. The least squares estimate [24], [25] of $\mathbf{g}_{n}$ is then given as

$$
\hat{\mathbf{g}}_{n}=\frac{\mathbf{y}_{n} \mathbf{S}^{H}}{P_{l}}=\sigma_{n} \mathbf{g}_{n}+\frac{\mathbf{n}_{n} \mathbf{S}^{H}}{P_{l}} .
$$

Equation (4) shows that error row vector $\mathbf{e}_{d n}=\frac{\mathbf{n}_{n} \mathbf{S}^{H}}{P_{l}} \in \mathbb{C}^{N}$ is independent of $\mathbf{g}_{n}$, and its entries are iid with $\operatorname{pdf} \mathcal{C} \mathcal{N}\left(0, \frac{N_{0}}{P_{l}}\right)$. This implies that in the estimated downlink channel matrix $\widehat{\mathbf{G}}=\left[\begin{array}{lll}\hat{\mathbf{g}}_{1}^{T} & \cdots & \hat{\mathbf{g}}_{2 M}^{T}\end{array}\right]^{T}=\mathbf{D}^{\frac{1}{2}} \mathbf{G}+\mathbf{E}_{d}$, the error matrix $\mathbf{E}_{d}$ has entries independent of the downlink channel matrix $\mathbf{G}=$ $\left[\begin{array}{lll}\mathbf{g}_{1}^{T} & \cdots & \mathbf{g}_{2 M}^{T}\end{array}\right]^{T}$, where $\mathbf{E}_{d}=\left[\begin{array}{lll}\mathbf{e}_{d 1}^{T} & \cdots & \mathbf{e}_{d 2 M}^{T}\end{array}\right]^{T}$.

\section{PRECODER DESIGN}

We begin this section by defining the structure of precoder $\mathbf{F}$, wherein we decompose it as $\mathbf{F}=\mathbf{F}_{\mathrm{d}} \mathbf{F}_{\mathrm{p}} \mathbf{F}_{\mathrm{u}}$, where $\mathbf{F}_{\mathrm{u}} \in$ $\mathbb{C}^{2 M \times N}$ is designed to mitigate the uplink MAC-phase MUI from the relay's received signal $\mathbf{y}_{r}$ for large $N$. To achieve this objective, we design $\mathbf{F}_{\mathrm{u}}$ based on the MRC criteria using the UL channel estimated by the relays i.e. set $\mathbf{F}_{\mathrm{u}}=\widehat{\mathbf{H}}^{H} \in$ 
$\mathbb{C}^{2 M \times N}$. The precoder is set to $\mathbf{F}_{\mathrm{p}}=\operatorname{blk-\operatorname {diag}}\left\{\mathbf{P}_{1}, \cdots, \mathbf{P}_{M}\right\}$, where $\mathbf{P}_{i}=\left[\begin{array}{lll}0 & 1 ; 1 & 0\end{array}\right]$ is a $2 \times 2$ permutation matrix which ensures that the symbol $x_{2 i-1}$ transmitted by the $(2 i-1)$ th user in the MAC phase is sent to the intended $2 i$ th user in the BC phase. Finally, the DL MRT precoder

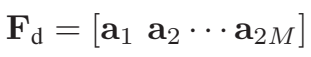

is designed for mitigating the DL BC-phase MUI for large $N$. The column vector $\mathbf{a}_{i^{\prime}}=\left[a_{1 i^{\prime}}, \cdots, a_{N i^{\prime}}\right]^{T} \in \mathbb{C}^{N}$ is the precoding vector for the $i^{\prime}$ th receiver. The entries of $\mathbf{a}_{i^{\prime}}$ are $a_{k i^{\prime}}=e^{j \theta_{k i^{\prime}}}$ for $k=1$ to $N$ transmit relay antennas, and for $i^{\prime}=1$ to $2 M$ users. The signal received by the $i^{\prime}$ th user in (2) can be expressed by substituting the expression of the precoder $\mathbf{F}$ in (2) as

$$
y_{i^{\prime}}=\underbrace{c \sigma_{i^{\prime}} \sigma_{i} \mathbf{g}_{i^{\prime}} \mathbf{a}_{i^{\prime}} \hat{\mathbf{h}}_{i}^{H} \mathbf{h}_{i} x_{i}}_{\text {Desired signal }}+\tilde{R}_{I_{i^{\prime}}}+\tilde{N}_{I_{i^{\prime}}}+n_{i^{\prime}} .
$$

The term $\tilde{R}_{I_{i^{\prime}}}$ is the sum of the MUI and the SI experienced by the $i^{\prime}$ th user due to its MAC-phase transmit signal $x_{i^{\prime}}$. The term $\tilde{R}_{I_{i^{\prime}}}$ can be expressed as $\tilde{R}_{I_{i^{\prime}}}$

$$
\begin{aligned}
& =c \sigma_{i^{\prime}} \mathbf{g}_{i^{\prime}} \mathbf{a}_{i^{\prime}} \sum_{m=1, m \neq i}^{2 M} \sigma_{m} \hat{\mathbf{h}}_{i}^{H} \mathbf{h}_{m} x_{m} \\
& +\sum_{l^{\prime}=1, l^{\prime} \neq i^{\prime}}^{2 M} c \sigma_{i^{\prime}} \mathbf{g}_{i^{\prime}} \mathbf{a}_{l^{\prime}} \sum_{m=1}^{2 M} \sigma_{m} \hat{\mathbf{h}}_{l}^{H} \mathbf{h}_{m} x_{m} \\
& =\underbrace{c \sigma_{i^{\prime}} \mathbf{g}_{i^{\prime}} \mathbf{a}_{i^{\prime}} \sigma_{i^{\prime}} \hat{\mathbf{h}}_{i}^{H} \mathbf{h}_{i^{\prime}} x_{i^{\prime}}+\sum_{l^{\prime}=1, l^{\prime} \neq i^{\prime}}^{2 M} c \sigma_{i^{\prime}} \mathbf{g}_{i^{\prime}} \mathbf{a}_{l^{\prime}} \sigma_{i^{\prime}} \hat{\mathbf{h}}_{l}^{H} \mathbf{h}_{i^{\prime}} x_{i^{\prime}}}_{\text {MUI }} \\
& +\underbrace{c \sigma_{i^{\prime}} \mathbf{g}_{i^{\prime}} \mathbf{a}_{i^{\prime}} \sum_{m=1, m \neq i, i^{\prime}}^{2 M} \sigma_{m} \hat{\mathbf{h}}_{i}^{H} \mathbf{h}_{m} x_{m}}_{\text {SI }} \\
& +\underbrace{\sum_{l^{\prime}=1, l^{\prime} \neq i^{\prime}}^{2 M} c \sigma_{i^{\prime}} \mathbf{g}_{i^{\prime}} \mathbf{a}_{l^{\prime}} \sum_{m}^{2 M} \sigma_{m} \hat{\mathbf{h}}_{l}^{H} \mathbf{h}_{m} x_{m}}_{m=1, m \neq i^{\prime}} .
\end{aligned}
$$

Since user $i^{\prime}$ does not have the knowledge of the UL channel $\sigma_{i^{\prime}} \mathbf{h}_{i^{\prime}}$, it cannot cancel the SI term. The term $\tilde{N}_{I_{i^{\prime}}}$ denotes the MAC-phase thermal noise forwarded by the relay to the $i^{\prime}$ user node, and is given as

$$
\tilde{N}_{I_{i^{\prime}}}=c \sigma_{i^{\prime}} \sum_{l^{\prime}=1}^{2 M} \mathbf{g}_{i^{\prime}} \mathbf{a}_{l^{\prime}} \tilde{n}_{l}, \text { where } \tilde{n}_{l}=\hat{\mathbf{h}}_{l}^{H} \mathbf{n}_{r}
$$

We assume that $x_{i}$ is from an $M$-QAM constellation, and use $\triangle x_{i}$ to denote the minimum distance across all pairs of constellation points. For a given channel realization, the noise- free minimum received distance squared at the $i^{\prime}$ th user is

$$
\begin{aligned}
& d_{i^{\prime}}^{2}=c^{2} \sigma_{i^{\prime}}^{2} \sigma_{i}^{2}\left|\mathbf{g}_{i^{\prime}} \mathbf{a}_{i^{\prime}} \hat{\mathbf{h}}_{i}^{H} \mathbf{h}_{i} \triangle x_{i}\right|^{2}, \text { where } \\
& \mathbf{g}_{i^{\prime}} \mathbf{a}_{i^{\prime}} \hat{\mathbf{h}}_{i}^{H} \mathbf{h}_{i} \triangle x_{i} \\
&=\left(\sum_{n=1}^{N} g_{i^{\prime} n} e^{j \theta_{n i^{\prime}}}\right)\left(\sum_{n=1}^{N} \hat{h}_{n i}^{*} h_{n i}\right) \\
&=\triangle x_{i}\left(\sum_{n=1}^{N}\left|g_{i^{\prime} n}\right| e^{j\left(\alpha_{i^{\prime} n}+\theta_{n i^{\prime}}\right)}\right)\left(\sum_{n=1}^{N} \hat{h}_{n i}^{*} h_{n i}\right) \\
&=\Delta x_{i}\left(\sum_{n=1}^{N}\left|g_{i^{\prime} n}\right| e^{j \delta_{n i^{\prime}}^{\left[i^{\prime} n\right]}}\right)\left(\sum_{n=1}^{N} \hat{h}_{n i}^{*} h_{n i}\right) .
\end{aligned}
$$

In the above equations, $g_{i^{\prime} n}=\left|g_{i^{\prime} n}\right| e^{j \alpha_{i^{\prime} n}}$. We define

$$
\delta_{n i^{\prime}}^{[i l]}=\alpha_{i l}+\theta_{n i^{\prime}},
$$

where the superscript $[i l]$ and subscript $n i^{\prime}$ denote the indices of $\alpha_{i l}$ and $\theta_{n i^{\prime}}$, respectively.

The symbol-PEP is determined by the ratio [26]

$$
\frac{d_{i^{\prime}}^{2}}{\left|\tilde{R}_{I_{i^{\prime}}}\right|^{2}+\left|\tilde{N}_{I_{i^{\prime}}}\right|^{2}+N_{0}} .
$$

We will show that for the MRC-MRT precoder, both the interference power $\left|\tilde{R}_{I_{i^{\prime}}}\right|^{2}$ and the forwarded noise power $\left|\tilde{N}_{I_{i^{\prime}}}\right|^{2}$ almost surely go to zero as $N \rightarrow \infty$. Consequently, the symbol PEP asymptotically approaches that of an AWGN channel. For a given $B \geq 1$, we assign the precoder phase angles $\theta_{n i^{\prime}}$ from a uniform quantizer as shown below

$$
\begin{aligned}
& \theta_{n i^{\prime}}=-\pi+(k-1) \frac{2 \pi}{2^{B}}=-\mu_{S_{k}}, \text { if } \hat{\alpha}_{i^{\prime} n} \in S_{k}, \text { where } \\
& S_{k}=\left[\pi-\left(k-\frac{1}{2}\right) \frac{2 \pi}{2^{B}}, \pi-\left(k-\frac{3}{2}\right) \frac{2 \pi}{2^{B}}\right),
\end{aligned}
$$

and $\mu_{S_{k}}$ is the centroid of $S_{k}$ and

$$
[\widehat{\mathbf{G}}]_{i^{\prime} n}=\hat{g}_{i^{\prime} n}=\left|\hat{g}_{i^{\prime} n}\right| e^{j \hat{\alpha}_{i^{\prime} n}} .
$$

We observe that the intervals $S_{i}$ and $S_{j}$ are disjoint for $i \neq j$ and $\bigcup_{i=1}^{2^{B}} S_{i}=[-\pi, \pi)$.

Channel feedback: The $n$th user quantizes the phases of its estimated downlink channel $\hat{\mathbf{g}}_{n}$ spanning from the relay and feeds back its index (see (13) and (14)) to the relay using a low-rate feedback channel. The relay uses these indices to construct the downlink transmit precoder $\mathbf{F}_{d}$ (see (5)). Such quantization techniques are extensively used for reducing channel feedback requirements in conventional MIMO systems [26], as well as in the commercial cellular systems, such as LTE-A [27].

Remark 2: We note that the zero-forcing receive/zeroforcing transmit (ZFR/ZFT) precoder has better rate for given $N$ and $M$. But we also note that the ZFR/ZFT precoder has $O\left(N^{3}\right)$ complexity, which is much higher than the MRC/MRT complexity of $O(2 M N)$. In this work we investigate the BER of the low-complexity quantized MRC/MRT precoder. It is important to investigate the BER of the high-rate quantized ZFR/ZFT precoder also, which can be taken up as future work. 
Remark 3: The MRC-MRT precoder in (5) has $2 M$ columns of the $N$-length vector $\mathbf{a}_{i^{\prime}}=\left[\begin{array}{lll}e^{j \theta_{i^{\prime}, 1}} & \ldots & e^{j \theta_{i^{\prime}, N}}\end{array}\right]^{T}$. Now if $\widehat{\mathbf{G}}$ is the downlink channel from the relay to the $2 M$ users and its entries are $[\widehat{\mathbf{G}}]_{i^{\prime}, n}=\left|\hat{g}_{i^{\prime}, n}\right| e^{j \hat{\alpha}_{i^{\prime}, n}}$, then each $\theta_{n, i^{\prime}}$, for $B$ quantized bits, takes values from $2^{B}$ levels which are defined in (13) and (14). The above design rule allows the phases $\theta_{n, i^{\prime}}$ to take the complex conjugates of the phases of the channel coefficients $[\widehat{\mathbf{G}}]_{i^{\prime}, n}=\left|\hat{g}_{i^{\prime}, n}\right| e^{j \hat{\alpha}_{i^{\prime}, n}}$.

We use a uniform quantizer since it minimizes the BER degradation over all quantizers, when the channel phases are iid and have a uniformly distributed pdf in $[-\pi, \pi)$ [28]. Having defined the precoder $\mathbf{F}$, we simplify $\mathbb{E}\left[\left\|\mathbf{F} \mathbf{y}_{r}\right\|^{2}\right]$ in (1), which will be useful for our analysis in the sequel. It is fairly easy to show that $\mathbb{E}\left[\left\|\mathbf{F} \mathbf{y}_{r}\right\|^{2}\right]$

$$
\begin{aligned}
& =\mathbb{E}\left[\operatorname{Tr}\left(\mathbf{F}_{\mathrm{d}} \mathbf{F}_{\mathrm{p}} \widehat{\mathbf{H}}^{H} \mathbf{y}_{r} \mathbf{y}_{r}^{H} \widehat{\mathbf{H}} \mathbf{F}_{\mathrm{p}} \mathbf{F}_{\mathrm{d}}^{H}\right)\right] \\
& =\mathbb{E}\left[\operatorname{Tr}\left(\mathbf{F}_{\mathrm{p}} \mathbf{F}_{\mathrm{d}}^{H} \mathbf{F}_{\mathrm{d}} \mathbf{F}_{\mathrm{p}} \widehat{\mathbf{H}}^{H} \mathbf{y}_{r} \mathbf{y}_{r}^{H} \widehat{\mathbf{H}}\right)\right] \\
& =\operatorname{Tr}\left(\mathbf{F}_{\mathrm{p}} \mathbb{E}\left[\mathbf{F}_{\mathrm{d}}^{H} \mathbf{F}_{\mathrm{d}}\right] \mathbf{F}_{\mathrm{p}} \mathbb{E}\left[\widehat{\mathbf{H}}^{H} \mathbf{y}_{r} \mathbf{y}_{r}^{H} \widehat{\mathbf{H}}\right]\right) \\
& =N \operatorname{Tr}\left(\mathbf{I}_{2 M} \mathbb{E}\left[\widehat{\mathbf{H}}^{H} \mathbf{y}_{r} \mathbf{y}_{r}^{H} \widehat{\mathbf{H}}\right]\right) \\
& =P_{s} N^{3}\left(\sum_{i=1}^{2 M} \sigma_{i}^{4}\right)+N^{2}\left(\sum_{i=1}^{2 M} \sigma_{i}^{2}\right)^{2}+P_{s} N^{2}(2 M) \eta_{u}^{2}\left(\sum_{i=1}^{2 M} \sigma_{i}^{2}\right) \\
& +N_{0} N^{2}\left(\sum_{i=1}^{2 M} \sigma_{i}^{2}\right)+N_{0} N^{2}(2 M) \eta_{u}^{2} .
\end{aligned}
$$

\section{BOUND ON THE NUMBER OF USERS TO ACHIEVE AGWN-LIKE PERFORMANCE}

In this section, we analyze the PEP, the per-user rate and the EE of the quantized MRC-MRT precoder design by varying the number of users $2 M$ with relay antennas $N$ i.e., $2 M=$ $\left\lfloor N^{\alpha}\right\rfloor$.

\section{A. Characterization of the MUI and SI power experienced by user $i^{\prime}$}

Our objective is to find a bound on $\alpha$ so that $\left|\tilde{R}_{I_{i^{\prime}}}\right|^{2} \stackrel{\text { a.s. }}{\rightarrow} 0$ as both $N$ and the number of users $2 M=\left\lfloor N^{\alpha}\right\rfloor$ tend to infinity. We do this in the following steps.

1) Using Markov's inequality [20], we find the bound $P\left(\left|\tilde{R}_{I_{i}}\right|^{K}>\epsilon\right)$ as well as $P\left(\left|\tilde{N}_{I_{i}}\right|^{K}>\epsilon\right)$ for $\epsilon>0$ and for an even natural number $K$.

2) We show that for $\alpha \in[0,1)$ and $P_{r}=\frac{2 M E_{r}}{N}$, the sums $\sum_{N=1}^{\infty} P\left(\left|\tilde{R}_{I_{i^{\prime}}}\right|^{K}>\epsilon\right), \sum_{N=1}^{\infty} P\left(\left|\tilde{N}_{I_{i^{\prime}}}\right|^{K}>\epsilon\right)$ are finite.

3) We invoke the First Borel-Cantelli Lemma [20] to show that both $\tilde{R}_{I_{i^{\prime}}}$ and later $\tilde{N}_{I_{i^{\prime}}}$, almost surely tend to zero.

4) We use the almost sure limit results on continuous functions to get $\frac{1}{\left|\tilde{R}_{I_{i^{\prime}}}\right|^{2}+\left|\tilde{N}_{I_{i^{\prime}}}\right|^{2}+N_{0}} \stackrel{a . s .}{\rightarrow} \frac{1}{N_{0}}$ for $N \rightarrow \infty$.

Let us now state Markov's inequality and the First BorelCantelli Lemma from [20].

Lemma 4.1: Markov's inequality: For a non-negative random variable $X$, we have:

$$
P(X>\epsilon) \leq \frac{\mathbb{E}[X]}{\epsilon}, \text { for given } \epsilon>0
$$

Lemma 4.2: First Borel-Cantelli Lemma: Let $A_{n}$ represent events obeying $\sum_{n=1}^{\infty} P\left(A_{n}\right)<\infty$. Then almost surely only finite number of ${ }^{n} \bar{A}_{n}^{1}$ events will occur.

Now, for even $K$, we have:

$$
\begin{aligned}
& P\left(\left|\tilde{R}_{I_{i}}\right|^{K}>\epsilon\right) \stackrel{(a)}{\leq} \frac{\mathbb{E}\left[\left|\tilde{R}_{I_{i}}\right|^{K}\right]}{\epsilon} \\
& \stackrel{(b)}{<} O\left[(2 M)^{\frac{K}{2}} N^{\frac{3 K}{2}}\right] \frac{\tilde{k} c^{K} \sigma_{i^{\prime}}^{K}}{\epsilon}+O\left[(2 M)^{K} N^{K}\right] \frac{\tilde{\tilde{k}} c^{K} \sigma_{i^{\prime}}^{K}}{\epsilon} \\
& \stackrel{(c)}{<} \frac{O\left[(2 M)^{\frac{K}{2}} N^{\frac{3 K}{2}}\right] \tilde{k} P_{r}^{\frac{K}{2}} \sigma_{u}^{K}}{\left(P_{s} N^{3} 2 M \sigma_{l}^{4}\right)^{\frac{K}{2}} \epsilon}+\frac{O\left[(2 M)^{K} N^{K}\right] \tilde{\tilde{k}} P_{r}^{\frac{K}{2}} \sigma_{u}^{K}}{\left(P_{s} N^{3} 2 M \sigma_{l}^{4}\right)^{\frac{K}{2}} \epsilon} \\
& =\frac{O[1] \tilde{k} P_{r}^{\frac{K}{2}} \sigma_{u}^{K}}{\left(P_{s} \sigma_{l}^{4}\right)^{\frac{K}{2}} \epsilon}+\frac{O\left[(2 M)^{\frac{K}{2}} N^{\frac{-K}{2}}\right] \tilde{\tilde{k}} P_{r}^{\frac{K}{2}} \sigma_{u}^{K}}{\left(P_{s} \sigma_{l}^{4}\right)^{\frac{K}{2}} \epsilon} \\
& \stackrel{(d)}{=} \frac{\tilde{\tilde{k}} E_{r}^{\frac{K}{2}} \sigma_{u}^{K}}{N^{\frac{K-\alpha K}{2}}\left(P_{s} \sigma_{l}^{4}\right)^{\frac{K}{2}} \epsilon}+\frac{N^{K-\alpha K}\left(P_{s} \sigma_{l}^{4}\right)^{\frac{K}{2}} \epsilon}{N^{K}}
\end{aligned}
$$

where

- Inequality $(a)$ follows from Markov's inequality.

- Inequality (b) follows from Appendix A, where we show that for a given number of feedback bits $B \geq 1$, even $K$ and large $N, 2 M$, the interference term $\tilde{R}_{I_{i^{\prime}}}$ defined in (7), obeys the bound $\frac{\mathbb{E}\left[\mid \tilde{R}_{\left.\left.I_{i^{\prime}}\right|^{K}\right]} c^{K} \sigma_{i^{\prime}}^{K}\right.}{2}<O[(2 M-$ $\left.1)^{\frac{K}{2}} N^{\frac{3 K}{2}}\right] \tilde{k}+O\left[(2 M-1)^{K}(N)^{K}\right] \tilde{\tilde{k}}$, where $\tilde{\tilde{k}}$ is some constant. The method of expanding higher moments of random variables using the $O[\cdot]$ notation is well illustrated by Tao [19].

- Equality $(c)$ is true because from (1) and (16), we have,

$$
c \leq \sqrt{\frac{P_{r}}{P_{s} N^{3} \sum_{i=1}^{2 M} \sigma_{i}^{4}}} \leq \sqrt{\frac{P_{r}}{P_{s} 2 M N^{3} \sigma_{l}^{4}}},
$$

where $\sigma_{l}=\inf \left\{\sigma_{1} \cdots \sigma_{2 M}\right\}$ and $\sigma_{u}=\sup \left\{\sigma_{1} \cdots \sigma_{2 M}\right\}$.

- Equality in $(d)$ is true because of the relay power allocation of $P_{r}=\frac{2 M E_{r}}{N}$ in the presence of $2 M=\left\lfloor N^{\alpha}\right\rfloor$ users. This choice of $P_{r}$, as derived later in Section IV-C, ensures the convergence of the received signal distance square to a non-zero constant, as $N \rightarrow \infty$.

From (18), we have

$$
\begin{aligned}
& \sum_{N=1}^{\infty} P\left(\left|\tilde{R}_{I_{i^{\prime}}}\right|^{K}>\epsilon\right) \\
& <\sum_{N=1}^{N^{\prime}} P\left(\left|\tilde{R}_{I_{i^{\prime}}}\right|^{K}>\epsilon\right)+\frac{\tilde{k} E_{r}^{\frac{K}{2}} \sigma_{u}^{K}}{\left(P_{s} \sigma_{l}^{4}\right)^{\frac{K}{2}} \epsilon} \sum_{N=N^{\prime}}^{\infty} \frac{1}{N^{\frac{K-\alpha K}{2}}} \\
& +\frac{\tilde{\tilde{k}} E_{r}^{\frac{K}{2}} \sigma_{u}^{K}}{\left(P_{s} \sigma_{l}^{4}\right)^{\frac{K}{2}}} \sum_{N=N^{\prime}}^{\infty} \frac{1}{N^{K-\alpha K}} .
\end{aligned}
$$

The tail series only obey $\sum_{N=N^{\prime}}^{\infty} \frac{1}{N^{\frac{K-\alpha K}{2}}}<\infty$ when $\frac{K-\alpha K}{2}>$ 1 , since $\sum_{N=1}^{\infty} \frac{1}{N^{p}}$ converges for $p>1$. Likewise, $\sum_{N=N^{\prime}}^{\infty} \frac{1}{N^{K-\alpha K}}$ is finite only when $K-\alpha K>1$. This implies that (20) is only finite when $\alpha<1-\frac{2}{K}$. We see that if we fix $K$ to a large enough value, when $N \rightarrow \infty$, the condition simplifies 
to $\alpha<1$. This is possible, since all even- $K$ moments of the Gaussian random variable exist and are finite.

Remark 4: Note that a sufficient condition for the summation in (20) to be finite is that the lower bound of the large scale fading parameters must be non-zero i.e., $\sigma_{l}>0$ and the upper bound be finite i.e., $\sigma_{u}<\infty$. The authors of [12], [13] do not impose such conditions on the large scale fading parameters, since the number of users in [12], [13] does not scale with the number of relay antennas $N$.

By using the First Borel-Cantelli lemma (see Lemma 4.2), we therefore have $\tilde{R}_{I_{i^{\prime}}} \stackrel{a . s .}{\rightarrow} 0$ as $N \rightarrow \infty$, for $\alpha \in[0,1)$. Since $|x|^{2}$ is continuous in $x$, invoking the Continuous Mapping Theorem of [20], we have

$$
\left|\tilde{R}_{I_{i^{\prime}}}\right|^{2} \stackrel{a . s .}{\rightarrow} 0 \text { as } N \rightarrow \infty .
$$

Both the MUI and SI terms almost surely go to zero for $P_{r}=$ $\frac{2 M E_{r}}{N}$, and the number of users $2 M=\left\lfloor N^{\alpha}\right\rfloor$ with $\alpha<1$, for any arbitrary choice of feedback bits $B \geq 1$ and channel estimation error variance, as $N \rightarrow \infty$.

Remark 5: The sum in (20) is finite for $\alpha<1$, because all the even moments of a Gaussian random variable exist. Let us suppose that instead of Gaussian, the channel entries are iid with some zero-mean, complex-valued distribution. Then for this new distribution, it turns out that the derivation of (18) and (20) remains exactly the same. Also, at this point let us assume that all higher moments beyond $K$ th moment do not exist for the new distribution. Then (20) is finite only when $\alpha<1-\frac{2}{K}$ implying the the number of users can only grow as fast $N^{1-\frac{2}{K}}$. This implies that the above analysis characterizing the MUI and SI is applicable for any zero-mean non-Gaussian complex distribution with the pdf $f_{\alpha_{i^{\prime} n}}($.$) of \alpha_{i^{\prime} n}$ satisfying $f_{\alpha_{i^{\prime} n}}(x)=$ $f_{\alpha_{i^{\prime} n}}(x+\pi)$, as shown in Appendix $B$. We make another crucial observation. For the Gaussian case, with $\alpha=0$ and $B=\infty$, our analysis reduces to that of [12], [13], where the authors fixed the number of users $2 M$, while increasing the number of relay antennas.

B. Characterization of the noise forwarded by the relay to user $i^{\prime}$

The forwarded noise term from (8) can be expressed as

$$
\begin{aligned}
& \tilde{N}_{I_{i^{\prime}}} \\
& =c \sigma_{i^{\prime}} \sum_{l^{\prime}=1}^{2 M} \mathbf{g}_{i^{\prime}} \mathbf{a}_{l^{\prime}} \tilde{n}_{l}=c \sigma_{i^{\prime}}\left(\mathbf{g}_{i^{\prime}} \mathbf{a}_{i^{\prime}} \tilde{n}_{i}+\sum_{l^{\prime}=1, l^{\prime} \neq i}^{2 M} \mathbf{g}_{i^{\prime}} \mathbf{a}_{l^{\prime}} \tilde{n}_{l}\right) \\
& =c \sigma_{i^{\prime}}\left(\sum_{p=1}^{N}\left|g_{i^{\prime} p}\right| e^{j \delta_{p i^{\prime}}^{\left[i^{\prime} p\right]}} \tilde{n}_{i}+\sum_{l^{\prime}=1, l^{\prime} \neq i}^{2 M} \sum_{p^{\prime}=1}^{N}\left|g_{i^{\prime} p^{\prime}}\right| e^{j \delta_{p^{\prime} l^{\prime}}^{\left[i^{\prime} p^{\prime}\right]}} \tilde{n}_{l}\right),
\end{aligned}
$$

where $\tilde{n}_{l}=\hat{\mathbf{h}}_{l}^{H} \mathbf{n}_{r}$. By using Markov's inequality we have

$$
\begin{aligned}
& P\left(\left|\tilde{N}_{I_{i^{\prime}}}\right|^{K}>\epsilon\right) \leq \frac{\mathbb{E}\left[\left|\tilde{N}_{I_{i^{\prime}}}\right|^{K}\right]}{\epsilon} \\
& \stackrel{(a)}{<}\left(O\left[N^{\frac{3 K}{2}}\right]+O\left[(2 M)^{\frac{K}{2}} N^{K}\right)\right] \frac{c^{K} \sigma_{i^{\prime}}^{K} \tilde{k}}{\epsilon} \\
& \stackrel{(b)}{<}\left(O\left[N^{\frac{3 K}{2}}\right]+O\left[(2 M)^{\frac{K}{2}} N^{K}\right)\right] \frac{P_{r}^{\frac{K}{2}} \sigma_{u}^{K} \tilde{k}}{\left(P_{s} 2 M N^{3} \sigma_{l}^{4}\right)^{\frac{K}{2}} \epsilon}
\end{aligned}
$$

$$
\begin{aligned}
& \stackrel{(c)}{=}\left(O\left[N^{\frac{3 K}{2}}\right]+O\left[(2 M)^{\frac{K}{2}} N^{K}\right]\right) \frac{E_{r}^{\frac{K}{2}} \sigma_{u}^{K} \tilde{k}}{\left(P_{s} N^{4} \sigma_{l}^{4}\right)^{\frac{K}{2}} \epsilon} \\
& \stackrel{(d)}{=} \frac{E_{r}^{\frac{K}{2}} \sigma_{u}^{K} \tilde{k}}{N^{\frac{K}{2}}\left(P_{s} \sigma_{l}^{4}\right)^{\frac{K}{2}} \epsilon}+\frac{E_{r}^{\frac{K}{2}} \sigma_{u}^{K} \tilde{k}}{N^{K-\frac{\alpha K}{2}}\left(P_{s} \sigma_{l}^{4}\right)^{\frac{K}{2}} \epsilon} .
\end{aligned}
$$

- Inequality in $(a)$ follows from Appendix C, where we show that for even $K$, and for large $N$ and $2 M$, the relay noise term $\tilde{N}_{I_{i^{\prime}}}$ can be bounded as $\frac{\mathbb{E}\left[\left|\tilde{N}_{I_{i^{\prime}}}\right|^{K}\right]}{c^{K} \sigma_{i^{\prime}}^{K}}<O\left[N^{\frac{3 K}{2}}\right] \tilde{k}+$ $O\left[(2 M)^{\frac{K}{2}} N^{K}\right] \tilde{k}$.

- Inequality in (b) follows from (19) and from the fact that $\sigma_{u} \geq \sigma_{i} \forall i$.

- Equality in $(c)$ follows the assignment $P_{r}=\frac{2 M E_{r}}{N}$.

- Equality in $(d)$ is true, because $2 M=\left\lfloor N^{\alpha}\right\rfloor$.

Now, from (23) we have,

$$
\begin{aligned}
& \sum_{N=1}^{\infty} P\left(\left|\tilde{N}_{I_{i^{\prime}}}\right|^{K}>\epsilon\right) \\
& <\sum_{N=1}^{N^{\prime}} P\left(\left|\tilde{N}_{I_{i^{\prime}}}\right|^{K}>\epsilon\right)+\sum_{N=N^{\prime}}^{\infty} \frac{E_{r}^{\frac{K}{2}} \sigma_{u}^{K} \tilde{k}}{N^{\frac{K}{2}}\left(P_{s} \sigma_{l}^{4}\right)^{\frac{K}{2}} \epsilon} \\
& +\sum_{N=N^{\prime}}^{\infty} \frac{E_{r}^{\frac{K}{2}} \sigma_{u}^{K} \tilde{k}}{N^{K-\frac{\alpha K}{2}}\left(P_{s} \sigma_{l}^{4}\right)^{\frac{K}{2}} \epsilon} .
\end{aligned}
$$

For large $K$ and $\alpha<1$, the tail series $\sum_{N=N^{\prime}}^{\infty} \frac{1}{N^{\frac{K}{2}}}$ and $\sum_{N=N^{\prime}}^{\infty} \frac{1}{N^{K-\frac{\alpha K}{2}}}$ in (24) are finite and consequently $\sum_{N=1}^{\infty} P\left(\left|\tilde{N}_{I_{i^{\prime}}}\right|^{K}>\epsilon\right)<\infty$. Hence, by the Borel-Cantelli Lemma (see Lemma 4.2), the noise term $\tilde{N}_{I_{i^{\prime}}} \stackrel{\text { a.s. }}{\rightarrow} 0$ as $N \rightarrow$ $\infty$, for $\alpha<1$. Since $|x|^{2}$ is continuous in $x \in \mathbb{C}$, we have,

$$
\left|\tilde{N}_{I_{i^{\prime}}}\right|^{2} \stackrel{\text { a.s. }}{\rightarrow} 0 \text { for } \alpha<1 \text { as } N \rightarrow \infty \text {. }
$$

From (21) and (25), and for $\alpha \in[0,1), 2 M=\left\lfloor N^{\alpha}\right\rfloor$ and $P_{r}=\frac{2 M E_{r}}{N}$, the term $\left|\tilde{R}_{I_{i^{\prime}}}\right|^{2}+\left|\tilde{N}_{I_{i^{\prime}}}\right|^{2} \stackrel{\text { a.s. }}{\rightarrow} 0$ as $N \rightarrow \infty$. We therefore have,

$$
\frac{1}{\left|\tilde{R}_{I_{i^{\prime}}}\right|^{2}+\left|\tilde{N}_{I_{i^{\prime}}}\right|^{2}+N_{0}} \stackrel{\text { a.s. }}{\rightarrow} \frac{1}{N_{0}} \text { as } N \rightarrow \infty .
$$

The above result follows from the fact that the term $\frac{1}{x+N_{0}}$ is continuous in $x \in \mathbb{C}$ for a fixed $N_{0}>0$ and the convergence of the term $\left(\left|\tilde{R}_{I_{i^{\prime}}}\right|^{2}+\left|\tilde{N}_{I_{i^{\prime}}}\right|^{2}\right)$ is in almost sure sense.

Remark 6: Note that (20) and (24) are not finite for $K=$ $\infty$. Hence $\alpha=1$ is not achievable with the aid of MRC-MRT scheme.

Remark 7: Equation (18) shows that the rate of convergence of $\tilde{R}_{I^{\prime}}$ to zero does not depend on any particular $\left(i^{\prime}, i\right)$ receiver-transmitter pair. Hence, $\tilde{R}_{I_{i^{\prime}}}$ converges uniformly to 0 for all users. Likewise, $\tilde{N}_{I_{i^{\prime}}}$ converges uniformly to 0 for all users. This fact will be used in Appendix D. 
C. Characterization of the received distance squared for user $i^{\prime}$ and relay power scaling

We know from (9) and (10) that the received distance at the $i^{\prime}$ th user is given by

$$
d_{i^{\prime}}=\left|\triangle x_{i}\right| c \sigma_{i^{\prime}} \sigma_{i}\left(\sum_{n=1}^{N}\left|g_{i^{\prime} n}\right| e^{j \delta_{n i^{\prime}}^{\left[i^{\prime}\right]}}\right)\left(\sum_{n=1}^{N} \hat{h}_{n i}^{*} h_{n i}\right) .
$$

For $2 M=\left\lfloor N^{\alpha}\right\rfloor$ and $\alpha \in[0,1)$, by substituting $c=$ $\sqrt{\frac{P_{r}}{\mathbb{E}\left[\left\|\mathbf{F} \mathbf{y}_{r}\right\|^{2}\right]}}$ from (16) and introducing the short hand

$k_{N}=\sqrt{\frac{\frac{N P_{r}}{N^{\alpha}}}{\frac{P_{s} \sum_{i=1}^{N^{\alpha}} \sigma_{i}^{4}}{N^{\alpha}}+\frac{\left(\sum_{i=1}^{N^{\alpha}} \sigma_{i}^{2}\right)^{2}}{N^{1+\alpha}}+\frac{P_{s} \eta_{u}^{2} \sum_{i=1}^{N^{\alpha}} \sigma_{i}^{2}}{N}+\frac{N_{0} \sum_{i=1}^{N^{\alpha}} \sigma_{i}^{2}}{N^{1+\alpha}}+\frac{N_{0} \eta_{u}^{2}}{N}}}$

we get $d_{i^{\prime}}$

$$
\begin{aligned}
& =k_{N}\left|\triangle x_{i}\right| \sigma_{i^{\prime}} \sigma_{i} \frac{\sum_{n=1}^{N}\left|g_{i^{\prime} n}\right| e^{j \delta \delta_{n i^{\prime}}^{\left[i^{\prime} n\right]}}}{N} \frac{\sum_{n=1}^{N} \hat{h}_{n i}^{*} h_{n i}}{N} \\
& \stackrel{a . s .}{\rightarrow}\left|\triangle x_{i}\right| \sigma_{i^{\prime}} \mathbb{E}\left[\left|g_{i^{\prime} n}\right| e^{\left.j \delta_{n i^{\prime}}^{\left[i^{\prime} n\right]}\right]}\right] \mathbb{E}\left[\hat{h}_{n i}^{*} h_{n i}\right] \lim _{N \rightarrow \infty} k_{N} \\
& =\left|\triangle x_{i}\right| \sigma_{i^{\prime}} \mathbb{E}\left[\left|g_{i^{\prime} n}\right| e^{\left.j \delta_{n i^{\prime}}^{\left[i^{\prime} n\right]}\right]}\right] \mathbb{E}\left[\left(\sigma_{i} h_{n i}^{*}+\left[\mathbf{E}_{\mathrm{u}}\right]_{n i}^{*}\right) h_{n i}\right] \lim _{N \rightarrow \infty} k_{N} \\
& =\left|\triangle x_{i}\right| \sigma_{i^{\prime}} \sigma_{i}^{2} \mathbb{E}\left[\left|g_{i^{\prime} n}\right| e^{\left.j \delta \delta_{n i^{\prime}}^{\left[i^{\prime} n\right]}\right]} \sqrt{\frac{E_{r}}{P_{s} \lim _{N \rightarrow \infty} \frac{\sum_{i=1}^{N^{\alpha}} \sigma_{i}^{4}}{N^{\alpha}}}} .\right.
\end{aligned}
$$

- Equation (28) follows by applying SLLN to $\frac{\sum_{n=1}^{N}\left|g_{i^{\prime} n}\right| e^{j \delta \delta_{i^{\prime}}^{\left[i^{\prime} n\right]}}}{N}$ and $\frac{\sum_{n=1}^{N} \hat{h}_{n i}^{*} h_{n i}}{N}$ as $N \rightarrow \infty$.

- Relay power law: Equation (29) is true because $\lim _{N \rightarrow \infty} k_{N}=\sqrt{\frac{E_{r}}{P_{s} \lim _{N \rightarrow \infty} \frac{\sum_{i=1}^{N \alpha} \sigma_{i}^{4}}{N^{\alpha}}}}$, where $E_{r}=\frac{N P_{r}}{N^{\alpha}}$ for a constant $P_{s}$. For the received distance of user $i^{\prime}$ to be non-zero, we require $k_{N}>0$; consequently a constant $E_{r}$ is a possible choice. This implies that the relay power must scale as $P_{r}=\frac{N^{\alpha} E_{r}}{N}=\frac{2 M E_{r}}{N}$. This is different from the relay power laws derived in [12], [13] for TDD precoders, which do not account for the $N^{\alpha}$ term in $P_{r}$, which arises due to scaling the number of users as $2 M=N^{\alpha}$. This is because we simultaneously scale both $M$ and $N$ to $\infty$ whilst obeying $2 M=N^{\alpha}$. By contrast, the power scaling laws of [12], [13] are only valid for $N \rightarrow \infty$ in conjugation with a finite $M$, which does not allow them to vary simultaneously. Our current framework therefore allows us to investigate the BER, rate and energy efficiency, when both $M$ and $N$ are large. Now if we simultaneously vary both $M$ and $N$, we cannot use the law of large numbers to quantify the MUI term as in [12], [13]. This is explained as follows:

- Invoking strong law of large numbers (SLLN), the authors of [12], showed that the MUI term in (7) obeys $\tilde{R}_{I_{i}} \stackrel{a . s .}{\rightarrow} 0$ as $N \rightarrow \infty$ and for a fixed $2 M$. This is because according to SLLN for $N \rightarrow \infty$ the inner products between 1) uplink channel vectors $\hat{\mathbf{h}}_{i}^{H}$ and $\mathbf{h}_{m}$ for $i \neq m$; as well as 2) between the downlink channel vectors $\mathbf{g}_{i^{\prime}}$ and $\mathbf{a}_{l^{\prime}}$ for $i^{\prime} \neq l^{\prime}$ converge to zero in the expressions $\sum_{m=1, m \neq i}^{2 M} \sigma_{m} \hat{\mathbf{h}}_{i}^{H} \mathbf{h}_{m} x_{m}$ and $\sum_{l^{\prime}=1, l^{\prime} \neq i^{\prime}}^{2 M} c \sigma_{i^{\prime}} \mathbf{g}_{i^{\prime}} \mathbf{a}_{l^{\prime}} \sum_{m=1}^{2 M} \sigma_{m} \hat{\mathbf{h}}_{l}^{H} \mathbf{h}_{m} x_{m}$ in $\tilde{R}_{I_{i}}$ in (7) and consequently almost surely we have $\tilde{R}_{I_{i}} \rightarrow 0$. However, if the number of users varies with that of the relay antennas i.e., $2 M=\left\lfloor N^{\alpha}\right\rfloor$, the SLLN cannot be applied to each term in (7) i.e., to each term in the expression $\sum_{m=1, m \neq i}^{N^{\alpha}} \sigma_{m} \hat{\mathbf{h}}_{i}^{H} \mathbf{h}_{m} x_{m}$ and $\sum_{l^{\prime}=1, l^{\prime} \neq i^{\prime}}^{N^{\alpha}} c \sigma_{i^{\prime}} \mathbf{g}_{i^{\prime}} \mathbf{a}_{l^{\prime}} \sum_{m=1}^{N^{\alpha}} \sigma_{m} \hat{\mathbf{h}}_{l}^{H} \mathbf{h}_{m} x_{m}$ to conclude that $\tilde{R}_{I_{i}} \rightarrow$ 0 almost surely with $N \rightarrow \infty$, as the summation limit itself now varies with $N$.

- Similarly, for a fixed $2 M$, reference [12] approximates MUI $\tilde{R}_{I_{i}}$ by Gaussian noise for $N \gg 2 M$ by applying central limit theorem to each term in (7) and then adding up $2 M$ Gaussian terms. This simplifies the computation of the lower bound on the sum-rate. However if $2 M=\left\lfloor N^{\alpha}\right\rfloor$, this approximation cannot be applied to the individual terms.

In the current work, we overcome the above problems by using Markov's inequality (Lemma 4.1), Borel Cantelli Lemma (Lemma 4.2) and Dominated Convergence Theorem (Theorem 4.3), which allow us to derive power scaling laws which, are valid for a wide class of non-Gaussian channels channels whose higher moments do not exist (see Remark 5)and not only Gaussian channels as in [12], [13].

\section{Asymptotic pairwise error performance}

We now compute the symbol PEP, per-use rate and the system's EE in almost sure sense for large $N$ values. To derive this result, we will choose the following theorem proved in [20].

Theorem 4.3: Dominated Convergence Theorem: Let $X_{n}$ be a sequence of random variables so that for each $n$, $X_{n} \leq Y$ almost surely and $\mathbb{E}[|Y|]<\infty$. Then $\lim _{n \rightarrow \infty} \mathbb{E}\left[X_{n}\right]=$ $\mathbb{E}\left[\lim _{n \rightarrow \infty} X_{n}\right]$.

Note from (29), that the convergence in (29) is in almost sure sense, since $|x|^{2}$ is continuous in $\mathbb{C}$. Then we assert that

$$
d_{i^{\prime}}^{2} \stackrel{a . s .}{\rightarrow}\left(\sigma_{i}^{2} \sigma_{i^{\prime}}\left|\triangle x_{i}\right| \mathbb{E}\left[\left|g_{i^{\prime} n}\right| e^{\left.j \delta_{i^{\prime}}^{\left[i^{\prime} n\right.}\right]}\right]\right)^{2}\left(\frac{E_{r}}{P_{s} \lim _{N \rightarrow \infty} \frac{\sum_{i=1}^{N^{\alpha}} \sigma_{i}^{4}}{N^{\alpha}}}\right) .
$$

From (26) and (30), it follows that for $2 M=N^{\alpha}, P_{r}=$ $\frac{2 M E_{r}}{N}, \alpha<1$ and $N \rightarrow \infty$

$$
\frac{d_{i^{\prime}}^{2}}{\left|\tilde{R}_{I_{i^{\prime}}}\right|^{2}+\left|\tilde{N}_{I_{i^{\prime}}}\right|^{2}+N_{0}}
$$




$$
\stackrel{a . s .}{\longrightarrow} \frac{\left(\sigma_{i}^{2} \sigma_{i^{\prime}}\left|\Delta x_{i}\right| \mathbb{E}\left[\left|g_{i^{\prime} n}\right| e^{\left.j \delta_{n i^{\prime}}^{\left[i^{\prime} n\right.}\right]}\right]\right)^{2}}{N_{0}}\left(\frac{E_{r}}{P_{s} \lim _{N \rightarrow \infty} \frac{\sum_{i=1}^{N^{\alpha}} \sigma_{i}^{4}}{N^{\alpha}}}\right) .
$$

The asymptotic symbol-PEP of user $i^{\prime}$ averaged over all channel realizations is

$$
\begin{aligned}
& \lim _{N \rightarrow \infty} \mathbb{E}\left[Q\left(\sqrt{\frac{d_{i^{\prime}}^{2}}{\left|\tilde{R}_{I_{i^{\prime}}}\right|^{2}+\left|\tilde{N}_{I_{i^{\prime}}}\right|^{2}+N_{0}}}\right)\right] \\
& \stackrel{(a)}{=} \mathbb{E}\left[Q\left(\sqrt{\lim _{N \rightarrow \infty} \frac{d_{i^{\prime}}^{2}}{\left|\tilde{R}_{I_{i^{\prime}}}\right|^{2}+\left|\tilde{N}_{I^{\prime}}\right|^{2}+N_{0}}}\right)\right] \\
& \stackrel{(b)}{=} Q\left[\frac{\left|\triangle x_{i}\right| \sigma_{i^{\prime}} \sigma_{i}^{2} \mathbb{E}\left[\left|g_{i^{\prime} n}\right| e^{\left.j \delta_{n i^{\prime}}^{\left[i^{\prime} n\right]}\right]}\right.}{\sqrt{N_{0}}} \sqrt{\frac{E_{r}}{P_{s} \lim _{N \rightarrow \infty} \frac{\sum_{i=1}^{N^{\alpha}} \sigma_{i}^{4}}{N^{\alpha}}}}\right) \\
& \stackrel{(c)}{=} Q\left(\frac{\left|\triangle \tilde{x}_{i}\right| \zeta_{i, i^{\prime}} \sqrt{E_{r}}}{\sqrt{N_{0}}}\right) .
\end{aligned}
$$

Here the expectation is over all realizations of $\mathbf{H}, \mathbf{G}, \mathbf{E}_{\mathrm{u}}$ and $\mathbf{E}_{\mathrm{d}}$. The equality in (a) follows from Theorem 4.3, and because the complementary error function $Q(x) \leq 1$ and $Q(\sqrt{x})$ is non-negative and continuous in $x$ [29]. Equality in $(b)$ follows from (31). In $(c)$ we use the constant $\zeta_{i, i^{\prime}}=\frac{\sigma_{i^{\prime}} \sigma_{i}^{2} \mathbb{E}\left[\left|g_{i^{\prime} n}\right| e^{j \delta \delta_{i^{\prime}}}\right]}{\sqrt{\lim _{N \rightarrow \infty} \frac{\sum_{i=1}^{N \alpha} \sigma_{i}^{4}}{N \alpha}}}$. Since $\mathbb{E}\left[\left|x_{i}\right|^{2}\right]=P_{s}$, the term $\left|\triangle \tilde{x}_{i}\right|=\frac{\left|\triangle x_{i}\right|}{\sqrt{P_{s}}}$ represents the minimum distance among all the points of the unit-energy transmit constellation.

Remark 8: An AWGN channel is represented by $y=$ $\zeta_{i, i^{\prime}} x+n$, where $x$ is the transmit symbol with power $E_{r}$ and $n$ is iid $\mathcal{C N}\left(0, N_{0}\right)$, which has the same symbol PEP as that of (32). This verifies our claim that the per-user symbolPEP of the MRC-MRT precoder converges almost surely to that of an equivalent AWGN channel.

Remark 9: The impact of the DL channel estimation error and of the quantized precoder is captured in $\mathbb{E}\left[\left|g_{i^{\prime} n}\right| e^{j \delta_{n i^{\prime}}^{\left[i^{\prime} n\right]}}\right]$, which can be evaluated numerically. Note that the UL channel estimation error does not impact the asymptotic PEP, since (32) shows that the asymptotic PEP is not a function of the UL channel estimation error variance $\eta_{u}^{2}$.

Impact of the Number of Quantization Bits $(B)$ on Symbol-PEP: It is clear from (32) that $\mathbb{E}\left[\left|g_{i^{\prime} n}\right| e^{\left.j \delta_{n i^{\prime}}^{\left[i^{i} n\right]}\right]}\right.$ has to be computed to investigate the impact of quantization bits $B$ on the asymptotic per-user PEP. It is difficult to evaluate $\mathbb{E}\left[\left|g_{i^{\prime} n}\right| e^{j \delta_{n i^{\prime}}^{\left[i^{\prime} n\right]}}\right]$ to get any analytical result for the asymptotic PEP, when the estimation error $\eta_{u}^{2}$ and $\eta_{d}^{2}>0$. We now proceed to demonstrate how $B$ impacts the asymptotic PEP, when $\eta_{u}^{2}=\eta_{d}^{2}=0$.

We know from (11) that $\delta_{n i^{\prime}}^{\left[i^{\prime} n\right]}=\alpha_{i^{\prime} n}+\theta_{n i^{\prime}}$. Due to the precoding rule defined in (13) and (14) we can see that when $\eta_{u}^{2}=\eta_{d}^{2}=0$, we have $\delta_{n i^{\prime}}^{\left[i^{\prime} n\right]} \in\left[\frac{-\pi}{2^{B}}, \frac{\pi}{2^{B}}\right]$. For $(\beta, \beta+\delta \beta) \subset$
$\left[\frac{-\pi}{2^{B}}, \frac{\pi}{2^{B}}\right]$, we have,

$$
\begin{aligned}
& \left\{\delta_{n i^{\prime}}^{\left[i^{\prime} n\right]} \in(\beta, \beta+\delta \beta)\right\} \\
& =\bigcup_{k=1}^{2^{B}}\left\{\alpha_{i^{\prime} n} \in\left(\beta+\mu_{S_{k}}, \beta+\delta \beta+\mu_{S_{k}}\right)\right\} .
\end{aligned}
$$

Equation (33) is true because i) whenever $\alpha_{i^{\prime} n} \in(\beta+$ $\mu_{S_{k}}, \beta+\delta \beta+\mu_{S_{k}}$ ), the parameter obeys $\theta_{n i^{\prime}}=-\mu_{S_{k}}$ (see (13)); ii) $\alpha_{i^{\prime} n} \in\left(\beta+\mu_{S_{k}}, \beta+\delta \beta+\mu_{S_{k}}\right) \subset S_{k}$ for each $k=1, \cdots, 2^{B}$ (see (13), (14)); and iii) $\cup_{k=1}^{2^{B}} S_{k}=[-\pi, \pi]$ and $S_{k} \cap S_{l}=\phi, k \neq l$ (see text after (15)). Hence from (33), we have:

$$
\begin{aligned}
& P\left(\left\{\delta_{n i^{\prime}}^{\left[i^{\prime} n\right]} \in(\beta, \beta+\delta \beta)\right\}\right) \\
& =\sum_{k=1}^{2^{B}} P\left(\left\{\alpha_{i^{\prime} n} \in\left(\beta+\mu_{S_{k}}, \beta+\delta \beta+\mu_{S_{k}}\right)\right\}\right)=\frac{2^{B} \delta \beta}{2 \pi} .
\end{aligned}
$$

Equation (34) follows from the fact that the angle $\alpha_{i^{\prime} n}$ of the complex Gaussian random variable $g_{i^{\prime} n}$ is uniformly distributed in $[-\pi, \pi]$. Hence for $\eta_{u}^{2}=\eta_{d}^{2}=0, \delta_{n i^{\prime}}^{\left[i^{\prime} n\right]}$ is distributed uniformly with pdf $f_{\delta_{n i^{\prime}}^{\left[i^{\prime} n\right]}}(x)=\frac{2^{B}}{2 \pi}$, which is independent of $\left|g_{i^{\prime} n}\right|$, since $\alpha_{i^{\prime} n}$ is independent of $\left|g_{i^{\prime} n}\right|$. Note that $\left|g_{i^{\prime} n}\right|$ is Rayleigh distributed with a pdf of $f_{\left|g_{i^{\prime} n}\right|} \mid(y)=2 y e^{-y^{2}}$. Hence, we have:

$$
\begin{aligned}
& \mathbb{E}\left[| g _ { i ^ { \prime } n } | e ^ { j \delta _ { n i ^ { \prime } } ^ { [ i ^ { \prime } n ] } ] = } \mathbb { E } [ | g _ { i ^ { \prime } n } | ] \mathbb { E } \left[e^{\left.j \delta_{n i^{\prime}}^{\left[i^{\prime} n\right]}\right]}\right.\right. \\
& =\int_{0}^{\infty} y f_{\left|g_{i^{\prime} n}\right|}(y) d y \int_{\frac{-\pi}{2 B}}^{\frac{\pi}{2^{B}}} e^{j x} f_{\delta_{n i^{\prime}}^{\left[i^{\prime} n\right]}}(x) d x=\frac{\sqrt{\pi} \sin \left(\frac{\pi}{2^{B}}\right)}{\frac{2 \pi}{2^{B}}} .
\end{aligned}
$$

It follows from (32) and (35) that when $\eta_{u}^{2}=\eta_{d}^{2}=0$ the per-user asymptotic PEP is

$$
Q\left(\frac{\left|\triangle \tilde{x}_{i}\right| \sigma_{i^{\prime}} \sigma_{i}^{2} \sqrt{\pi} \sin \left(\frac{\pi}{2^{B}}\right) \sqrt{E_{r}}}{\sqrt{N_{0}} \frac{2 \pi}{2^{B}} \sqrt{\lim _{N \rightarrow \infty} \frac{\sum_{i=1}^{N^{\alpha}} \sigma_{i}^{4}}{N^{\alpha}}}}\right) .
$$

Remark 10: We see from (36) that the PEP of a quantized precoder is a function of $\frac{\sin \left(\frac{\pi}{2 B}\right)}{\frac{\pi}{2 B}}$, which has a maximum value of unity for $B=\infty$ and 0.9 for $B=2$. For $B=2$ the PEP, and consequently the BER, of the quantized MRC-MRT precoder will be close to that of the unquantized one for large $N$. We will validate this fact later in the numerical section. Although we do not have a closed-form PEP expression for analyzing the impact of quantization in presence of channel estimation error, we conjecture that with $B=2$ and $\eta_{u}^{2}$ and $\eta_{d}^{2}>0$, the BER of the quantized MRC precoder will be close to that of the unquantized one. We later numerically show that the conjecture is in fact true not only for the BER, but also for the per-user rate,EE.

Remark 11: From (36), we conclude that the smaller the parameter $\lim _{N \rightarrow \infty} \frac{\sum_{i=1}^{N^{\alpha}} \sigma_{i}^{4}}{N^{\alpha}}$, the better the asymptotic per-user PEP of the MRC-MRT precoder becomes. A smaller limit value 
implies larger path loss values on a average. This implies having a lower on average MUI power, leading to a better per-user BER.

\section{E. Asymptotic per-user rate}

The per-user rate is given by $\frac{1}{2} \mathbb{E}[\log (1+$ $\left.\left.\frac{p_{i^{\prime}}^{2}}{\left|\tilde{R}_{I_{i^{\prime}}}\right|^{2}+\left|\tilde{N}_{I_{i^{\prime}}}\right|^{2}+N_{0}}\right)\right]$, where $p_{i^{\prime}}^{2}=c^{2} \sigma_{i^{\prime}}^{2} \sigma_{i}^{2} P_{s}\left|\mathbf{g}_{i^{\prime}} \mathbf{a}_{i^{\prime}} \hat{\mathbf{h}}_{i}^{H} \mathbf{h}_{i}\right|^{2}$ is the instantaneous received signal power of user $i^{\prime}$. The $p_{i^{\prime}}^{2}$ expression is similar to the $d_{i}^{2}$ expression in (9). For $2 M=N^{\alpha}, P_{r}=\frac{2 M E_{r}}{N}$ and $\alpha \in[0,1)$, we can similarly prove that $\frac{p_{i^{\prime}}^{2}}{\left|\tilde{R}_{I_{i^{\prime}}}\right|^{2}+\left|\tilde{N}_{I_{i^{\prime}}}\right|^{2}+N_{0}}$

$$
\stackrel{\text { a.s. }}{\rightarrow} P_{s}\left(\sigma_{i}^{2} \sigma_{i^{\prime}} \mathbb{E}\left[\left|g_{i^{\prime} n}\right| e^{j \delta_{n i^{\prime}}^{\left[i^{\prime} n\right]}}\right]\right)^{2}\left(\frac{E_{r}}{P_{s} \lim _{N \rightarrow \infty} \frac{\sum_{i=1}^{N^{\alpha}} \sigma_{i}^{4}}{N^{\alpha}}}\right) \text {. }
$$

The asymptotic per-user rate is given by

$$
\begin{aligned}
& \lim _{N \rightarrow \infty} \frac{1}{2} \mathbb{E}\left[\log \left(1+\frac{p_{i^{\prime}}^{2}}{\left|\tilde{R}_{I_{i^{\prime}}}\right|^{2}+\left|\tilde{N}_{I_{i^{\prime}}}\right|^{2}+N_{0}}\right)\right] \\
& \stackrel{(a)}{\leq} \mathbb{E}\left[\lim _{N \rightarrow \infty} \frac{1}{2} \log \left(1+\frac{p_{i^{\prime}}^{2}}{\left|\tilde{R}_{I_{i^{\prime}}}\right|^{2}+\left|\tilde{N}_{I_{i^{\prime}}}\right|^{2}+N_{0}}\right)\right] \\
& \stackrel{(b)}{=} \frac{1}{2} \log \left(1+\frac{\left(\sigma_{i}^{2} \sigma_{i^{\prime}} \mathbb{E}\left[\left|g_{i^{\prime} n}\right| e^{\left.j \delta_{n i^{\prime}}^{\left[i^{\prime} n\right]}\right]}\right)^{2}\right.}{N_{0}}\left(\frac{E_{r}}{\lim _{N \rightarrow \infty} \frac{\sum_{i=1}^{N^{\alpha}} \sigma_{i}^{4}}{N^{\alpha}}}\right)\right) \\
& =\frac{1}{2} \log \left(1+\frac{E_{r} \zeta_{i, i^{\prime}}^{2}}{N_{0}}\right) .
\end{aligned}
$$

Inequality in step $(a)$ is from Fatou's lemma [20], which states that if a sequence of positive random variables $X_{n} \rightarrow X$ almost surely, then we have $\lim _{n \rightarrow \infty} \mathbb{E}\left[X_{n}\right] \leq \mathbb{E}[X]$. Equality in step (b) follows from (37).

Remark 12: We observe from (38) that for an arbitrary choice of $B \geq 1$, the asymptotic per-user rate is upper bounded by the rate of an AWGN channel $y=\zeta_{i, i^{\prime}} x+n$ with transmit power $E_{r}$ and noise $n$ having pdf $\mathcal{C N}\left(0, N_{0}\right)$ when i) the relay power is being scaled as $P_{r}=\frac{2 M E_{r}}{N}$; and ii) the number of users being scaled as $2 M=\left\lfloor N^{\alpha}\right\rfloor$ for $\alpha \in[0,1)$.

Impact of the Number of Quantization Bits $(B)$ on PerUser Rate: This can be observed by investigating (38), when the channel estimation error is $\eta_{u}^{2}=\eta_{d}^{2}=0$. Substituting the value of $\mathbb{E}\left[\left|g_{i^{\prime} n}\right| e^{j \delta_{n i^{\prime}}^{\left[i^{\prime} n\right]}}\right]$ from (35) in (38), we get an upper bound on the per-user rate as follows

$$
\frac{1}{2} \log \left(1+\frac{\sigma_{i}^{4} \sigma_{i^{\prime}}^{2} \pi \sin ^{2}\left(\frac{\pi}{2^{B}}\right) E_{r}}{N_{0}\left(\frac{2 \pi}{2^{B}}\right)^{2} \lim _{N \rightarrow \infty} \frac{\sum_{i=1}^{N^{\alpha}} \sigma_{i}^{4}}{N^{\alpha}}}\right) .
$$

We see from (39) that the per-user rate of a quantized precoder, similar to PEP, is a function of $\frac{\sin \left(\frac{\pi}{2^{B}}\right)}{\frac{\pi}{2^{B}}}$, which has a maximum value of unity for $B=\infty$ and 0.9 for $B=2$. Hence, for $B=2$ both quantized and unquantized MRC-MRT precoders will have similar rates, for large $N$, a fact which we will later validate in the numerical section.

\section{F. Asymptotic Energy efficiency}

Let us now investigate the EE of the quantized MRC-MRT precoder which is defined as $\rho_{N}=\frac{R_{N}}{P_{r}+P_{T}}$ [12]. Here $R_{n}$ is the system's sum rate, which is given by

$$
R_{N}=\frac{1}{2} \mathbb{E}\left[\sum_{i^{\prime}=1}^{2 M} \log \left(1+\frac{p_{i^{\prime}}^{2}}{\left|\tilde{R}_{I_{i^{\prime}}}\right|^{2}+\left|\tilde{N}_{I_{i^{\prime}}}\right|^{2}+N_{0}}\right)\right] .
$$

Furthermore, $P_{r}=\frac{2 M E_{r}}{N}$ is the total transmit power of the relay, and $P_{T}=2 M \mathbb{E}\left[\left|x_{i}\right|^{2}\right]=2 M P_{s}$ is the total transmit power of the $2 M$ single-antenna users. The asymptotic EE associated with $2 M=\left\lfloor N^{\alpha}\right\rfloor$ is given by

$$
\begin{aligned}
& \left.\lim _{N \rightarrow \infty} \rho_{N}=\lim _{N \rightarrow \infty} \frac{\sum_{i^{\prime}=1}^{N^{\alpha}} \mathbb{E}\left[\log \left(1+\frac{p_{i^{\prime}}^{2}}{\left|\tilde{R}_{I_{i^{\prime}}}\right|^{2}+\left|\tilde{N}_{I_{i^{\prime}}}\right|^{2}+N_{0}}\right)\right]}{\frac{2 N^{\alpha} E_{r}}{N}+2 N^{\alpha} P_{s}}\right] \\
& \stackrel{(a)}{\leq} \frac{1}{2 P_{s}} \mathbb{E}\left[\lim _{N \rightarrow \infty} \frac{\sum_{i^{\prime}=1}^{N^{\alpha}} \log \left(1+\frac{p_{i^{\prime}}^{2}}{\left|\tilde{R}_{i^{\prime}}\right|^{2}+\left|\tilde{N}_{I_{i^{\prime}}}\right|^{2}+N_{0}}\right)}{N^{\alpha}}\right] \\
& \stackrel{(b)}{=} \frac{1}{2 P_{s}} \lim _{N \rightarrow \infty} \frac{\sum_{i^{\prime}=1}^{N^{\alpha}} \log \left(1+\frac{E_{r} \zeta_{i, i^{\prime}}^{2}}{N_{0}}\right)}{N^{\alpha}}
\end{aligned}
$$

The inequality in step $(a)$ follows from Fatou's lemma [20]. Equality in $(b)$ is proved in Appendix D. The crucial step in proving the result in Appendix $D$ is to assume $\inf \left\{\sigma_{1}, \sigma_{2}, \ldots\right\}=\sigma_{l}>0$ and $\sup \left\{\sigma_{1}, \sigma_{2}, \ldots\right\}=\sigma_{u}<\infty$, which allows for uniform convergence of the interference powers for all the $\left(i, i^{\prime}\right)$ receiver transmitter pairs.

Impact of Quantization Bits $(B)$ on EE: The impact of quantization on asymptotic EE can be seen by investigating (41) when channel estimation error $\eta_{u}^{2}=\eta_{d}^{2}=0$. Similar to the derivation of (39), it can be shown that for $\eta_{u}^{2}=\eta_{d}^{2}=0$, the EE upper bound in (41) reduces to

$$
\frac{1}{2 P_{s}} \lim _{N \rightarrow \infty} \frac{\sum_{i^{\prime}=1}^{N^{\alpha}} \log \left(1+\frac{\sigma_{i}^{4} \sigma_{i^{\prime}}^{2} \pi \sin ^{2}\left(\frac{\pi}{2^{B}}\right) E_{r}}{N_{0}\left(\frac{2 \pi}{2^{B}}\right)^{2} \lim _{N \rightarrow \infty} \frac{\sum_{i=1}^{N^{\alpha}} \sigma_{i}^{4}}{N^{\alpha}}}\right)}{N^{\alpha}} .
$$

We see from (42) that EE of a quantized precoder, similar to the PEP and per-user rate, is a function of $\frac{\sin \left(\frac{\pi}{2^{B}}\right)}{\frac{\pi}{2^{B}}}$. Hence for $B=2$, and for large $N$, we will show that both quantized and unquantized MRC-MRT precoders will have similar EE.

\section{NumERICAL RESULTS AND DISCUSSIONS}

We now numerically investigate the BER, per-user rate and EE of the quantized MRC-MRT precoder using Monte Carlo simulations. We first investigate its BER and consider a system, where each user employs 4-QAM, and estimates its DL channel $\sigma_{i} \mathbf{g}_{i}$ and then uniformly quantizes phases of the 
entries of $\sigma_{i} \mathbf{g}_{i}$ using $B$ bits each. The relay also estimates the UL channel $\mathbf{H}$. We plot the BER of users versus its respective average receive SNR. For example the average receive SNR for user 1 is defined as $\frac{\mathbb{E}\left[\left|c \sigma_{1} \mathbf{g}_{1} \mathbf{a}_{1} \hat{\mathbf{h}}_{2}^{H} \sigma_{2} \mathbf{h}_{2} x_{2}\right|^{2}\right]}{\mathbb{E}\left[\left|n_{1}\right|^{2}\right]}$. It is worthwhile noting that whenever we vary the number of relay antenna $N$, we vary the number of users $2 M=\left\lfloor N^{\alpha}\right\rfloor$. This is in contrast to [12], where the number of users was fixed while varying $N$. Also, here we fix $\alpha=\frac{1}{3}$.

We begin our investigation by setting the large scale fading parameter $\sigma_{i}=1, \forall i=1, \cdots, 2 M$. Since all the users statistically observe the same channel, we plot the BER of first user alone. We recall from Remark 8 that for $\alpha \in[0,1)$ per-user symbol-PEP of the quantized MRC-MRT precoder converges almost surely to that of an equivalent AWGN channel $y=\zeta_{i, i^{\prime}} x+n$, where $\zeta_{i, i^{\prime}}$ is defined after (32). To verify this, we plot in Fig. 1a the BER of this equivalent AWGN channel versus the average receive SNR $\frac{\mathbb{E}\left[\left|\zeta_{i, i^{\prime}} x\right|^{2}\right]}{\mathbb{E}\left[|n|^{2}\right]}$ with $B=\infty$ and $\eta_{u}^{2}=\eta_{d}^{2}=0$. We observe that with $N=200$ and $B=2$, the first user BER is significantly inferior than the equivalent AWGN channel. With $N=1600, B=2$ and $2 M=12$, and at $10^{-4} \mathrm{BER}$, it requires only $1.6 \mathrm{~dB}$ higher SNR than that of the equivalent AWGN channel.

We now evaluate in Fig. 1a the effect of quantization on the BER of the MRC-MRT precoder using $B=1$ and $B=2$ bits. For benchmarking, we also plot the unquantized MRCMRT massive MIMO TWR relay precoder from [12], which is designed by assuming that the relay also has unquantized downlink CSI. We see that for $N=1600$ and $B=2$ bits, the BER of the quantized precoder is similar to that of the unquantized one. This can be justified by recalling that the asymptotic PEP, derived in (36), is a function of $\operatorname{sinc}\left(\frac{\pi}{2^{B}}\right)$. We now plot in Fig. $1 \mathrm{~b}$ the $\operatorname{sinc}\left(\frac{\pi}{2^{B}}\right)$ for different $B$ values. We see that for $B=2, \operatorname{sinc}\left(\frac{\pi}{2^{B}}\right)=\frac{\sin \left(\frac{\pi}{2^{B}}\right)}{\frac{\pi}{2^{B}}}=0.9$, which is $90 \%$ of its peak value. We note from Fig. 1 a that for $B=1$, the BER of the quantized precoder for $N=1600$ is $\approx 1.5 \mathrm{~dB}$ away from the unquantized one. This is because for $B=1, \operatorname{sinc}\left(\frac{\pi}{2^{B}}\right) \approx$ 0.65; the PEP expression in (36), and consequently the BER of the quantized precoder, proportionately degrades.

We now numerically show in Fig. 1c that the user PEP gracefully approaches the PEP of an equivalent AWGN channel with the number of relay antennas $N \rightarrow \infty$. For this study we have $2 M=N^{\frac{1}{3}}$, we fix $\eta_{u}^{2}=\eta_{d}^{2}=0$, and set $N_{0}=1$ and $B=2$. We observe that as $N$ increases from 8 to 1728 , the user PEP tends to its limit as calculated in (36). This figure thus confirms that the system at $N=1728$ for the aforementioned parameters starts experiencing asymptotic PEP behavior.

We now assume large scale fading parameters for different users are varied as $\sigma_{i}=d_{i}^{-2} 10^{\zeta_{i}}$ where $\zeta_{i}$ is iid with pdf $\mathcal{C N}(0,0.1)$ and $d_{i}$ varies uniformly between 0 to $2 \mathrm{kms}$ for $i=1, \cdots, 2 M$ [30]. We now plot the BER of the first three different users in Figs. 2a, 2b and 2c respectively for the following systems configuration: $N=200,1600$ relay antennas, $2 M=\left\lfloor N^{\frac{1}{3}}\right\rfloor$ users, and with channel estimation error variance $\eta_{u}^{2}=\eta_{d}^{2}=1$. We see from the plots that with $B=2$, the BER of all the three users with the quantized MRC-MRT precoder is close to the unquantized one. Also,

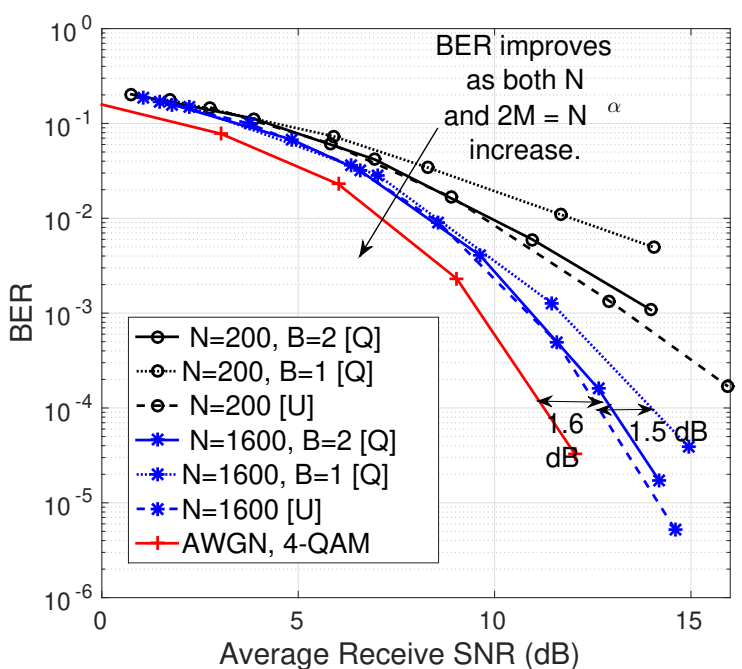

(a)

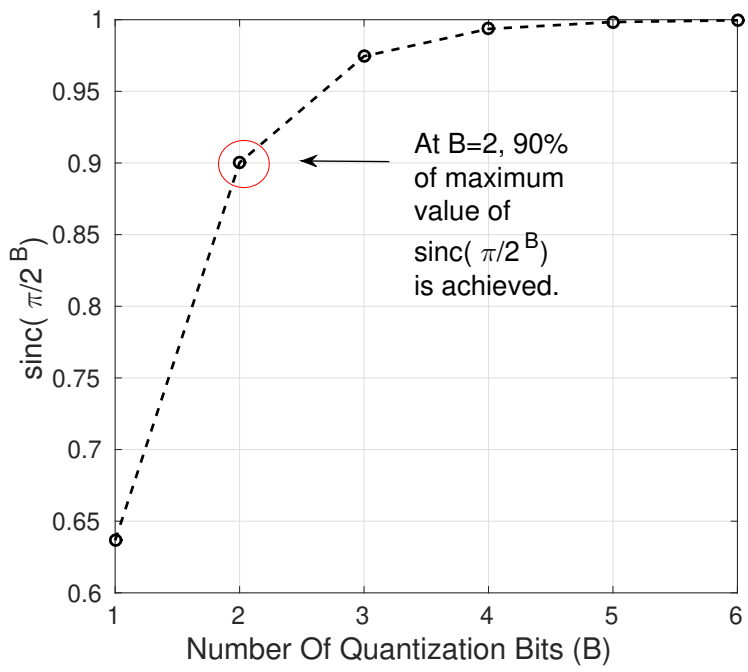

(b)

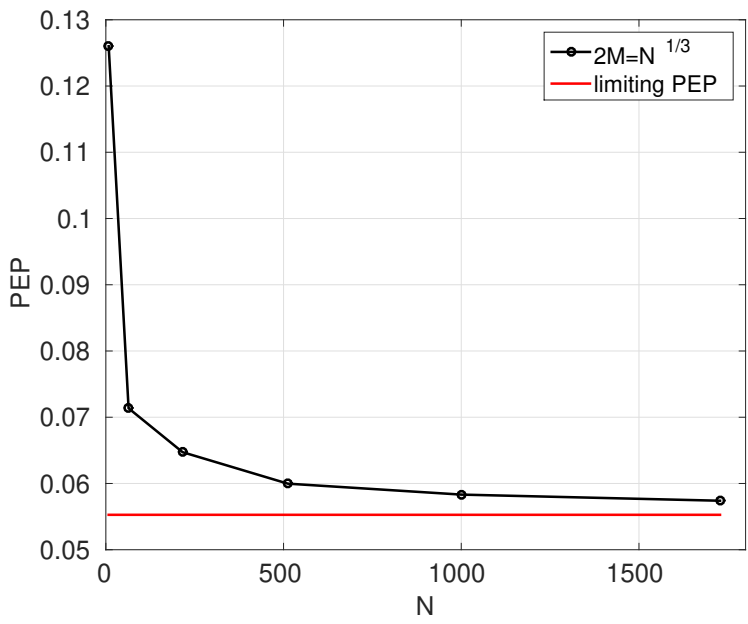

(c)

Fig. 1: a) BER of the quantized (Q) and unquantized (U) MRC-MRT precoder [12]. Also channel estimation error $\eta_{u}^{2}=$ $\eta_{d}^{2}=0$; (b) Variation of $\operatorname{sinc}\left(\frac{\pi}{2^{B}}\right)=\frac{\sin \left(\frac{\pi}{2^{B}}\right)}{\frac{\pi}{2^{B}}}$ with the number of quantization bits $B$; (c) Variation of averaged per-user PEP $\mathbb{E}_{H}\left[Q\left(\sqrt{\frac{d_{i^{\prime}}^{2}}{\left|\tilde{R}_{I_{i^{\prime}}}\right|^{2}+\left|\tilde{N}_{I^{\prime}}\right|^{2}+N_{0}}}\right)\right]$ with $N . N_{0}=1, P_{s}=E_{r}=2$. For Fig 1a) and 1c), each user employs 4-QAM, and the number of users $2 M$ varies with relay antennas $N$ as $2 M=\left\lfloor N^{\frac{1}{3}}\right\rfloor$. 


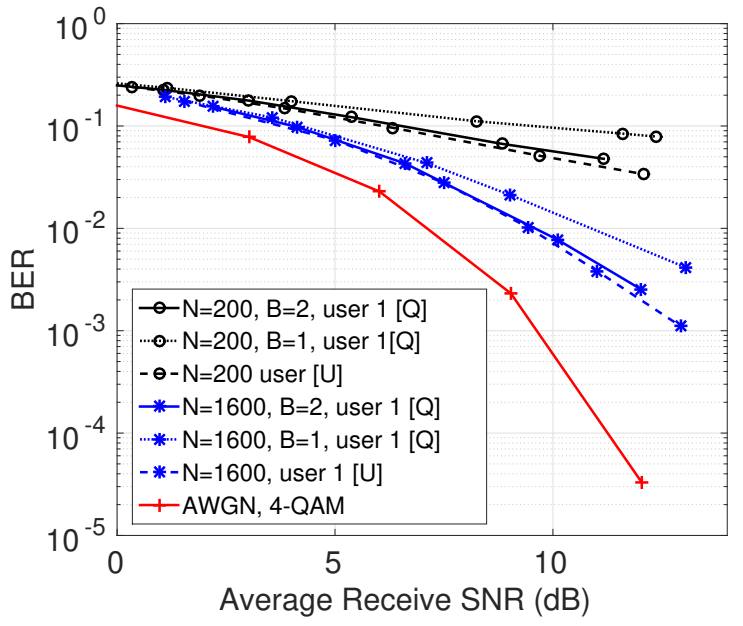

(a)

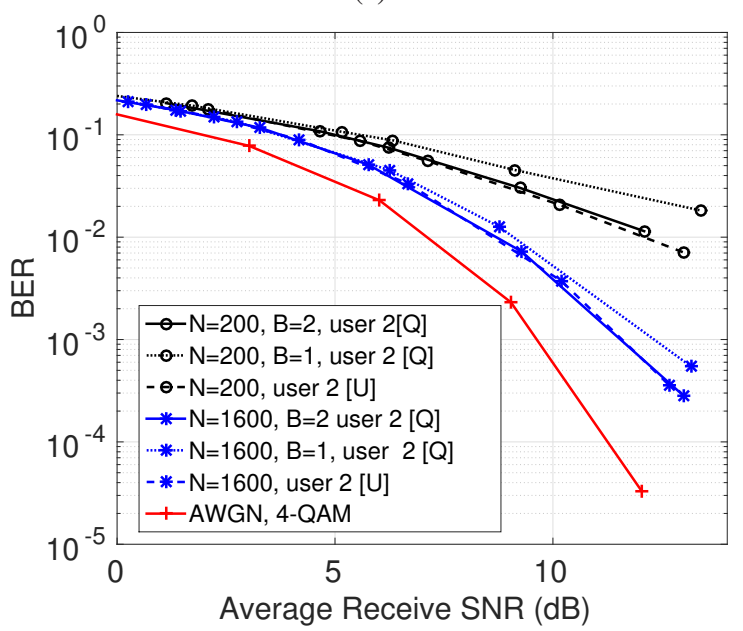

(b)

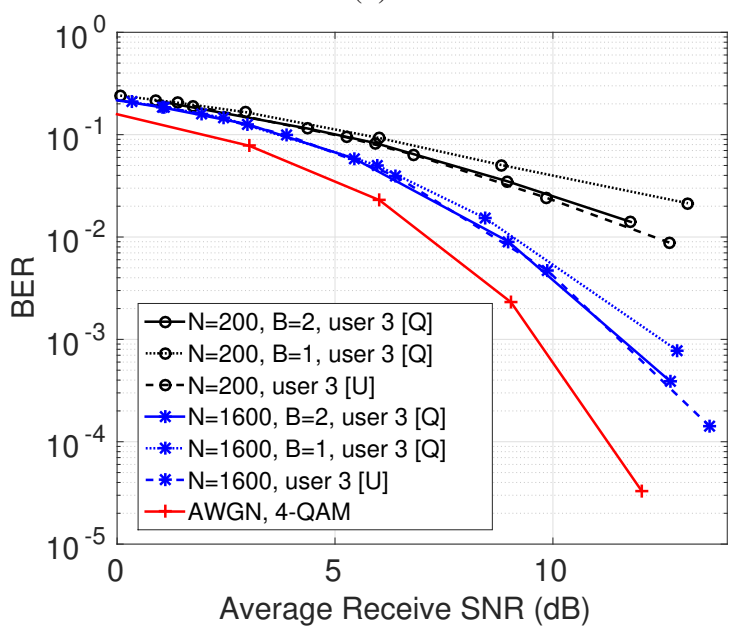

(c)

Fig. 2: BER of the quantized (Q) and unquantized MRC-MRT precoder [12] with non-unity large scale fading parameters for (a) User 1 and (b) User 2 and (c) User 3. The number of users $2 M$ varies with the number of relay antennas as $2 M=\left\lfloor N^{\frac{1}{3}}\right\rfloor$. We assume channel estimation error $\eta_{u}^{2}=\eta_{d}^{2}=1,4$-QAM modulation.
BER of each user reduces with increase in $N$.

We next plot the rate of the first three users of the quantized precoder in Figs. 3a, 3b and 3c by using the same system parameters as used in generating the plots in Fig. 2. We see from these figures that by increasing $N$ (and consequently the number of users $2 M=\left\lfloor N^{\alpha}\right\rfloor$ ), the per-user rate increases. We also see that the per-user rate, as also discussed in Remark 12, is upper-bounded by the rate of the equivalent AWGN channel $y=\zeta_{i, i^{\prime}} x+n$, where $\zeta_{i, i^{\prime}}$ is defined after (32). We also note that for every finite $N$, the per-user rate saturates beyond a certain SNR. This is because for every finite $N$, the MUI is strictly non-zero. Upon increasing $N$, the MUI of (21) tends to zero, which increases the rate. We also observe that for $B=2$, both the quantized and unquantized precoders have similar rates. For $B=1$, the quantized precoder, however, has a degraded performance. This is because the per-user rate upper-bound (39) is a function of $\operatorname{sinc}\left(\frac{\pi}{2^{B}}\right)$. For $B=2$, as shown earlier in Fig. 1b, $\operatorname{sinc}\left(\frac{\pi}{2^{B}}\right)$ achieves $90 \%$ percent of its maximum value, while for $B=1$ it achieves only $65 \%$ of its highest value, which degrades the per-user rate.

We next investigate the EE of the quantized MRC-MRT precoder. We commence with the case when the channel estimation error is $\eta_{u}^{2}=\eta_{d}^{2}=0$ and $\sigma_{i}=1 \forall i$. We observe in Fig. 4a that for $B=2$ and for large $N$, the $\mathrm{EE}$ of the quantized precoder is close to the unquantized one. For $B=1$, the EE however, degrades significantly. This is because, as shown in (42), for large $N$, the $\mathrm{EE}$ is a function of $\operatorname{sinc}\left(\frac{\pi}{2^{B}}\right)$. This term, as discussed earlier, for $B=2$ and $B=1$ achieves $90 \%$ and $65 \%$ percent of its maximum value, respectively. Furthermore, the EE increases with $N$ due to decrease in MUI (see (21)). Note that for $\sigma_{i}=1 \forall i$, the upper bound in (42) becomes $\frac{1}{2 P_{s}} \log \left(1+\frac{\pi E_{r} \sin ^{2}\left(\frac{\pi}{2 B}\right)}{N_{0}\left(\frac{2 \pi}{2 B}\right)^{2}}\right)$, which is the EE of an AWGN channel associated with $y=\frac{\sqrt{\pi} \sin \left(\frac{\pi}{2 B}\right) x}{\frac{2 \pi}{2 B}}+n$ as well as $\mathbb{E}\left[|x|^{2}\right]=E_{r}=P_{s}$ and $\mathbb{E}\left[|n|^{2}\right]=N_{0}$. We plot the EE of the AWGN channel for $B=\infty, E_{r}=P_{s}=2$ versus the average receive $S N R$ i.e., $\frac{\pi E_{r}}{4 N_{0}}$. We see from the figure that the EE of this AWGN channel upper bounds the EE of the quantized MRC-MRT precoder, a fact proved earlier in (42).

We next plot in Fig. 4b the EE for the channel estimation error variance of $\eta_{u}^{2}=\eta_{d}^{2}=1$ and the same large scale fading model as used in Fig. 2. We see for $B=2$ the $\mathrm{EE}$ of the quantized precoder is close to that of the unquantized one. This again validates the conjecture that $B=2$ is sufficient for the quantized MRC-MRT precoder to achieve a performance close to the unquantized one, even with channel estimation errors.

\section{Conclusions}

We investigated three performance metrics for quantized MRC/MRT precoder designed for FDD TWR, using $B \geq 1$ bits per transmit antenna per-user of the quantized DL channel information. We showed that the symbol-PEP, per-user rate and energy efficiency of this quantized MRC/MRT precoder converges almost surely to that of an AWGN channel by concurrently increasing the number of i) relay antennas $N \rightarrow \infty$; and ii) users as $2 M=\left\lfloor N^{\alpha}\right\rfloor$ with $\alpha \in[0,1)$. We showed 


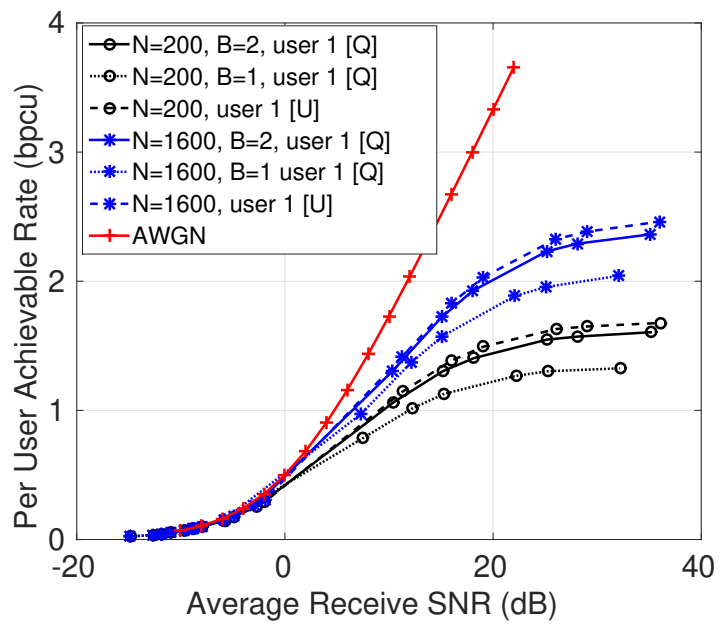

(a)

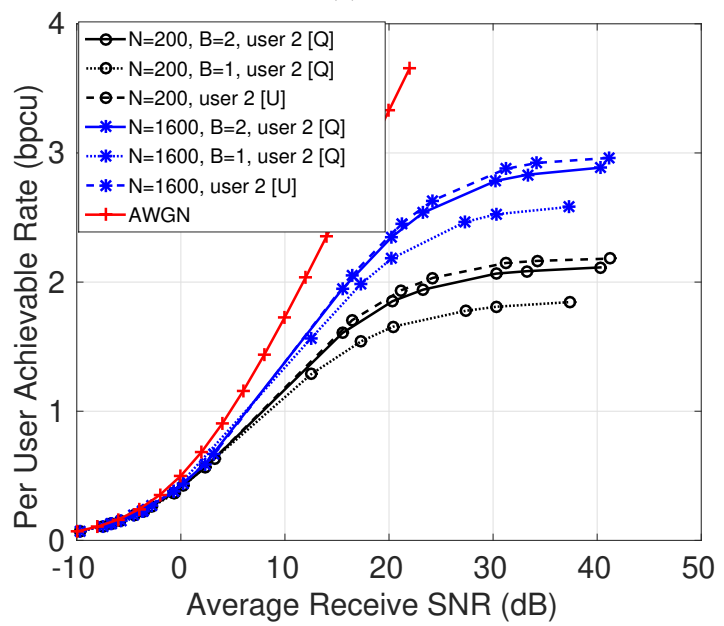

(b)

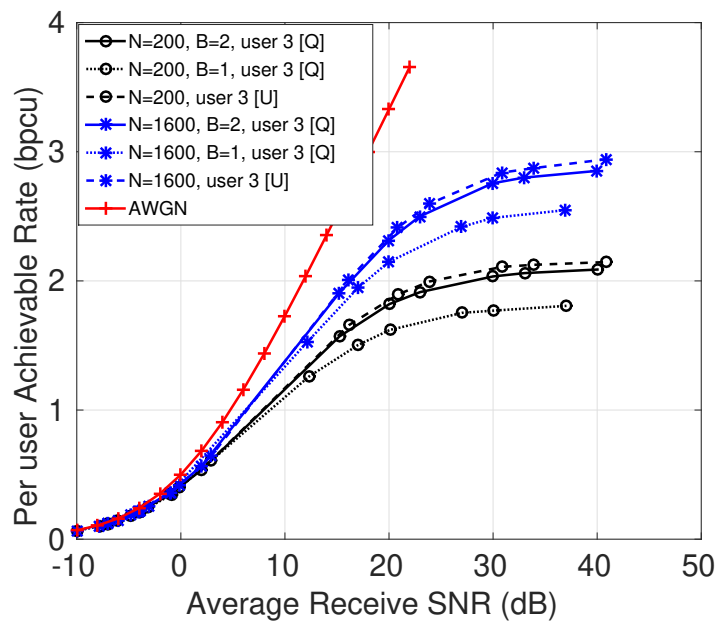

(c)

Fig. 3: Per user achievable rate comparison of the quantized (Q) and unquantized MRC-MRT precoder (from [12]) with non-unity large scale fading parameters for (a) User 1; (b) User 2 and (c) User 3. The number of users $2 M$ varies with the number of relay antennas $N=1600$ as $2 M=\left\lfloor N^{\frac{1}{3}}\right\rfloor$ and channel estimation error $\eta_{u}^{2}=\eta_{d}^{2}=$ 1.

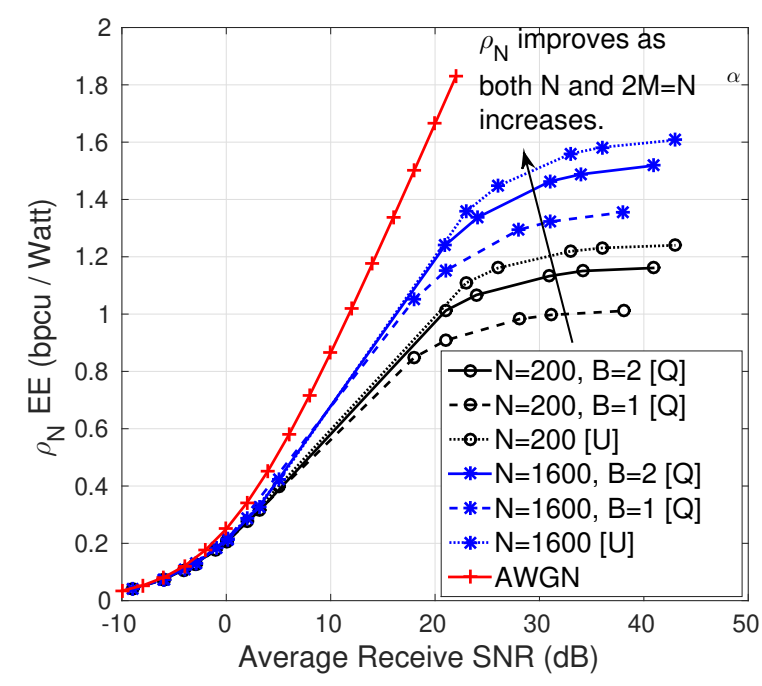

(a)

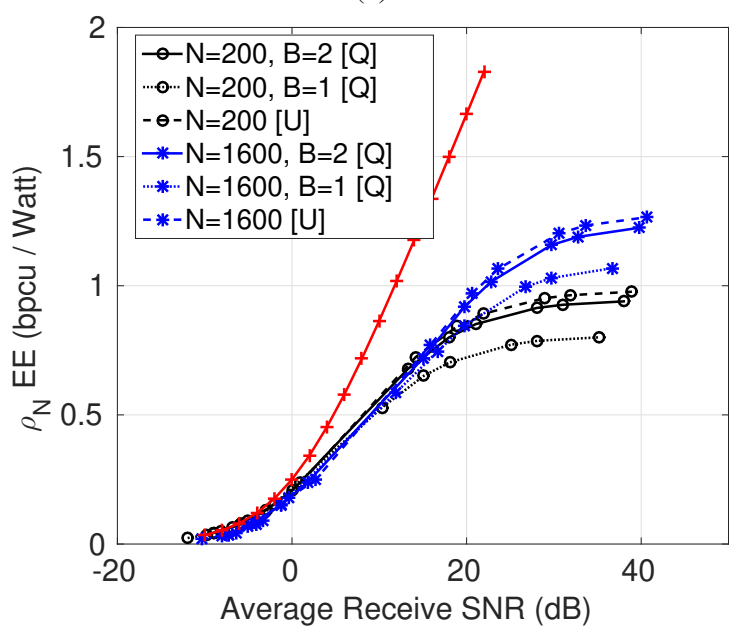

(b)

Fig. 4: Energy Efficiency (EE) comparison of the quantized(Q) and unquantized(U) MRC-MRT precoder (from [12]). The number of users $2 M$ varies with the number of relay antennas $N$ as $2 M=$ $\left\lfloor N^{\frac{1}{3}}\right\rfloor$ with (a) channel estimation error $\eta_{u}^{2}=\eta_{d}^{2}=0, \sigma_{i}=1 \forall i$; (b) $\eta_{u}^{2}=\eta_{d}^{2}=1, \sigma_{i} \neq 1$.

how to bound $\alpha$ when the channel coefficients are iid complex zero-mean non-Gaussian with a constraint on the pdf of the phase. The key conclusion from this work is that for $B=2$, the quantized MRC-MRT precoder performs very close to the unquantized MRC-MRT precoder in terms of BER, per-user rate and EE. This happens for large $N$, with the number of users scaled as $2 M=\left\lfloor N^{\alpha}\right\rfloor$, with $\alpha \in[0,1)$ with the relay power varied as $P_{r}=\frac{2 M E_{r}}{N}$ for a fixed per user transmit power $P_{s}$.

\section{ACKNOWLEDGEMENTS}

The authors would like to thank the editor Dr. Linglong Dai and the anonymous reviewers for their help in improving the quality of the paper. Rohit Budhiraja would like to acknowledge the financial support from Ministry of Electronics and Information Technology, Govt. of India, under Young Faculty Research Fellowship. L. Hanzo would like to acknowledge 
the financial support of the EPSRC projects EP/Noo4558/1, EP/PO34284/1, of the Royal Society's GRFC Grant as well as of the European Research Council's Advanced Fellow Grant QuantCom.

\section{APPENDIX A}

BOUND ON $\mathbb{E}\left[\left|\tilde{R}_{I_{i^{\prime}}}\right|^{K}\right]$

Claim: For large $N, 2 M$ and even $K$, the interference term $\tilde{R}_{I_{i^{\prime}}}$ defined in (7), satisfies

$$
\begin{aligned}
& \frac{\mathbb{E}\left[\left|\tilde{R}_{I_{i^{\prime}}}\right|^{K}\right]}{c^{K} \sigma_{i^{\prime}}^{K}} \\
& <O\left[(2 M-1)^{\frac{K}{2}} N^{\frac{3 K}{2}}\right] \tilde{k}+O\left[(2 M-1)^{K}(N)^{K}\right] \tilde{\tilde{k}} .
\end{aligned}
$$

Proof: Before proceeding further, we state and prove few lemmas.

Lemma A.1: For $l, l^{\prime} \in\{1 \cdots, i-1, i+1, \cdots, N\}$ and $n, p \in\{1, \cdots, N\}$ for $n \neq p$, random variables $\left|g_{i^{\prime} n}\right| e^{j \delta_{n l}^{\left[i^{\prime} n\right]}},\left|g_{i^{\prime} p}\right| e^{j \delta_{p l^{\prime}}^{\left[i^{\prime} p\right]}}$ are uncorrelated i.e., $\mathbb{E}\left[\left|g_{i^{\prime} n}\right| e^{j \delta_{n l}^{\left[i^{\prime} n\right]}}\left|g_{i^{\prime} p}\right| e^{-j \delta_{p l^{\prime}}^{\left[i^{\prime} p\right]}}\right]=0$ for a given $B \geq 1$.

Proof:

$$
\begin{aligned}
& \mathbb{E}\left[\left|g_{i^{\prime} n}\right| e^{j \delta_{n l}^{\left[i^{\prime} n\right]}}\left|g_{i^{\prime} p}\right| e^{-j \delta_{p l^{\prime}}^{\left[i^{\prime} p\right]}}\right] \\
& \stackrel{(a)}{=} \mathbb{E}\left[\left|g_{i^{\prime} n}\right| e^{j\left(\alpha_{i^{\prime} n}+\theta_{n l}\right)}\left|g_{i^{\prime} p}\right| e^{-j\left(\alpha_{i^{\prime} p}+\theta_{p l^{\prime}}\right)}\right] \\
& \stackrel{(b)}{=} \mathbb{E}\left[\left|g_{i^{\prime} n}\right|\right] \mathbb{E}\left[e^{j \alpha_{i^{\prime} n}}\right] \mathbb{E}\left[e^{j \theta_{n l}}\right] \mathbb{E}\left[\left|g_{i^{\prime} p}\right|\right] \mathbb{E}\left[e^{-j \alpha_{i^{\prime} p}}\right] \\
& \times \mathbb{E}\left[e^{\left.-j \theta_{p l^{\prime}}\right]}\right. \\
& \stackrel{(c)}{=} 0 .
\end{aligned}
$$

Equality in $(a)$ is due to the definitions of $\delta_{n l}^{\left[i^{\prime} n\right]}=\alpha_{i^{\prime} n}+$ $\theta_{n l}$ and $\delta_{p l^{\prime}}^{\left[i^{\prime} p\right]}=\alpha_{i^{\prime} p}+\theta_{p l^{\prime}}$. Equality in $(b)$ is because $\left|g_{i^{\prime} n}\right|,\left|g_{i^{\prime} p}\right|, \alpha_{i^{\prime} n}, \alpha_{i^{\prime} p}, \alpha_{l n}$ and $\alpha_{l^{\prime} p}$ are independent of each other, while $\theta_{n l}$ and $\theta_{p l^{\prime}}$ are solely a function of $\alpha_{l n}$ and $\alpha_{l^{\prime} p}$, respectively. Equality in $(c)$ is because as shown in Appendix B, we have $\mathbb{E}\left[e^{j \theta_{n l}}\right]=0$ for $B \geq 1$.

Lemma A.2: For $l \neq l^{\prime}$, the random variables $\left|g_{i^{\prime} n}\right| e^{j \delta_{n l}^{\left[i^{\prime} n\right]}}$ and $\left|g_{i^{\prime} n}\right| e^{j \delta_{n l^{\prime}}^{\left[i^{\prime} n\right]}}$ are uncorrelated i.e.,

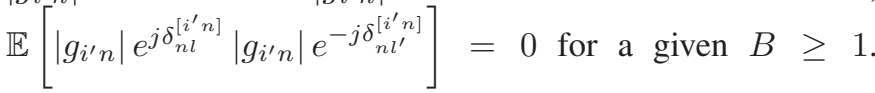

Proof: Similar to the proof of Lemma A.1.

The MUI term $\tilde{R}_{I_{i^{\prime}}}$ (7) is reformulated as (A.2a). Now, for an even $K$ we have

$$
\begin{aligned}
& \frac{\mathbb{E}\left[\left|\tilde{R}_{I_{i^{\prime}}}\right|^{K}\right]}{c^{K} \sigma_{i^{\prime}}^{K}} \\
& \stackrel{(a)}{=} \sum_{1 \leq i_{1}, \ldots, i_{K} \leq 2 M N} \mathbb{E}\left[X_{i_{1}} X_{i_{2}}^{*} \cdots X_{i_{K-1}} X_{i_{K}}^{*}\right]
\end{aligned}
$$

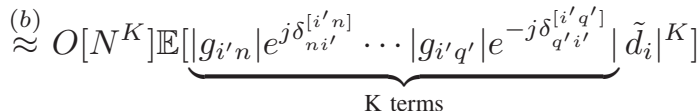

$$
\begin{aligned}
& +O\left[(2 M-1)^{\frac{K}{2}} N^{\frac{K}{2}}\right] \mathbb{E}[\underbrace{\left|g_{i^{\prime} n}\right|^{2}\left|\tilde{\tilde{d}}_{l}\right|^{2} \cdots\left|g_{i^{\prime} q}\right|^{2}\left|\tilde{\tilde{d}}_{l^{\prime}}\right|^{2}}_{\frac{K}{2} \text { terms }}] \\
& \stackrel{(c)}{=} O\left[N^{K}\right]\left(\mathbb { E } \left[\left|g_{i^{\prime} n}\right| e^{\left.\left.j \delta \delta_{n i^{\prime}}^{\left[i^{\prime} n\right]}\right]\right)^{K}} \mathbb{E}\left[\left|\tilde{d}_{i}\right|^{K}\right]\right.\right. \\
& +O\left[(2 M-1)^{\frac{K}{2}} N^{\frac{K}{2}}\right] \mathbb{E}\left[\left|g_{i^{\prime} n}\right|^{2} \cdots\left|g_{i^{\prime} q}\right|^{2}\right] \mathbb{E}\left[\left|\tilde{\tilde{d}}_{l}\right|^{2} \cdots\left|\tilde{\tilde{d}}_{l^{\prime}}\right|^{2}\right] \\
& \stackrel{(d)}{\leq} O\left[N^{K}\right] \mathbb{E}\left[\left|\tilde{d}_{i}\right|^{K}\right] \tilde{k}_{1} \\
& +O\left[(2 M-1)^{\frac{K}{2}} N^{\frac{K}{2}}\right] \mathbb{E}\left[\left|\tilde{\tilde{d}}_{l}\right|^{2} \cdots\left|\tilde{\tilde{d}}_{l^{\prime}}\right|^{2}\right] \tilde{k}_{1} .
\end{aligned}
$$

- Equality in $(a)$ is due to the $K$ th moment expansion formula in [19]. $X_{i}$ 's take the value $\left|g_{i^{\prime} n}\right| e^{j \delta_{n i^{\prime}}^{\left[i^{\prime} n\right]}} \tilde{d}_{i}$ from the first part of the sum in (A.2a) or $\left|g_{i^{\prime} n}\right| e^{j \delta_{n l^{\prime}}^{\left[i^{\prime} n\right]}} \tilde{\tilde{d}}_{l}$ from the second part of the sum in (A.2a). Further $\tilde{d}_{i}$, $\tilde{\tilde{d}}_{l}$ are defined as $\tilde{d}_{i}=\sum_{m=1, m \neq i}^{2 M} \sigma_{m} x_{m} \hat{\mathbf{h}}_{i}^{H} \mathbf{h}_{m}$ and $\tilde{d}_{l}=\sum_{m=1}^{2 M} \sigma_{m} x_{m} \hat{\mathbf{h}}_{l}^{H} \mathbf{h}_{m}$.

- The approximation in (b) lists the dominant terms in the expansion of step (a). Since, $\left|g_{i^{\prime} n}\right| e^{j \delta_{n i^{\prime}}^{\left[i^{\prime} n\right]}} \tilde{d}_{i}$ in the first part of (A.2a) are iid non-zero mean random variables, there can be $O\left[N^{K}\right]$ such terms as listed in step (b). Furthermore, $\left|g_{i^{\prime} n}\right| e^{j \delta_{n l^{\prime}}^{\left[i^{\prime} n\right]}} \tilde{\tilde{d}}_{l}$ in the second part of (A.2a) are zero mean iid random variables, so each $\left|g_{i^{\prime} n}\right| e^{j \delta_{n l^{\prime}}^{\left[i^{\prime} n\right]}} \tilde{\tilde{d}}_{l}$ must occur in pair along with its conjugate an even number of times in the expansion of the term in step (a), otherwise from Lemmas A.1 and A.2, their contribution will be zero. The dominant number of such terms is $O\left[(2 M-1)^{\frac{K}{2}} N^{\frac{K}{2}}\right]$ which happens when a typical $\left|g_{i^{\prime} n}\right| e^{j \delta_{n l^{\prime}}^{\left[i^{\prime} n\right]}} \tilde{\tilde{d}}_{l}$ term occurs exactly twice. Note that the approximation is tight for large $N$ and $2 M$ [19].

- Equality in $(c)$ is true, because entries of $\mathbf{G}, \mathbf{H}$ and $\mathbf{E}_{\mathrm{u}}, \mathbf{E}_{\mathrm{d}}$ are iid $\mathcal{C N}(0,1)$ and $\mathcal{C N}\left(0, \eta_{u}^{2}=\eta_{d}^{2}\right)$ respectively, and $\widehat{\mathbf{H}}=$ $\mathbf{H}+\mathbf{E}_{\mathrm{u}}$. This means that the entries of $\mathbf{G}$ are independent of $\widehat{\mathbf{H}}, \mathbf{H}$.

- Inequality in $(d)$ is true for a fixed $K$, because $\left(\mathbb{E}\left[\left|g_{i^{\prime} n^{\prime}}\right| e^{\left.\left.-j \delta_{n^{\prime} i^{\prime}}^{\left[i^{\prime} n^{\prime}\right]}\right]\right)^{K}}<\left(\mathbb{E}\left[\left|g_{i^{\prime} n^{\prime}}\right|^{2}\right]\right)^{\frac{K}{2}}<\infty\right.\right.$ (Cauchy-Swartz inequality) as the entries of $\mathbf{G}$ are iid $\mathcal{C N}(0,1)$. Similarly, $\mathbb{E}\left[\left|g_{i^{\prime} n}\right|^{2} \cdots\left|g_{i^{\prime} q}\right|^{2}\right]<\infty$ by the repeated application of the Cauchy Swartz inequality for a fixed $K$. Hence each of

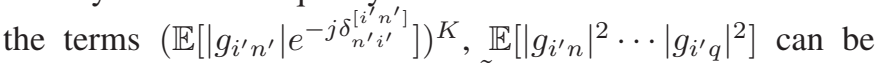
bounded by some finite constant $\tilde{k}_{1}$.

Equation (A.3) tells us that we have to compute $\mathbb{E}\left[\left|\tilde{d}_{i}\right|^{K}\right]$ and $\mathbb{E}\left[\tilde{\tilde{d}}_{l} \ldots \tilde{\tilde{d}}_{l^{\prime}}\right]$ to evaluate $\mathbb{E}\left[\left|\tilde{R}_{I_{i}}\right|^{K}\right]$. We next evaluate them as follows.

Lemma A.3: $\mathbb{E}\left[\left|\tilde{d}_{i}\right|^{K}\right]<O\left[(2 M-1)^{\frac{K}{2}} N^{\frac{K}{2}}\right] \tilde{k}_{2}$. 


$$
\begin{aligned}
& \frac{\tilde{R}_{I_{i^{\prime}}}}{c \sigma_{i^{\prime}}} \\
& =\left(\sum_{n=1}^{N}\left|g_{i^{\prime} n}\right| e^{j\left(\alpha_{i^{\prime} n}+\theta_{n i^{\prime}}\right)}\right)\left(\sum_{m=1, m \neq i}^{2 M} \sigma_{m} x_{m} \hat{\mathbf{h}}_{i}^{H} \mathbf{h}_{m}\right) \\
& +\sum_{l^{\prime}=1, l^{\prime} \neq i}^{2 M}\left\{\left(\sum_{n=1}^{N}\left|g_{i^{\prime} n}\right| e^{j\left(\alpha_{i^{\prime} n}+\theta_{n l^{\prime}}\right)}\right)\left(\sum_{m=1}^{2 M} \sigma_{m} x_{m} \hat{\mathbf{h}}_{l}^{H} \mathbf{h}_{m}\right)\right\} \\
& =\underbrace{\left(\sum_{n=1}^{N}\left|g_{i^{\prime} n}\right| e^{j \delta_{n i^{\prime}}^{\left[i^{\prime} n\right]}}\right)\left(\sum_{m=1, m \neq i}^{M} \sigma_{m} x_{m} \hat{\mathbf{h}}_{i}^{H} \mathbf{h}_{m}\right)}_{\text {part } 1}+\underbrace{\sum_{l^{\prime}=1, l^{\prime} \neq i}^{2 M}\left\{\left(\sum_{n=1}^{N}\left|g_{i^{\prime} n}\right| e^{j \delta_{n l^{\prime}}^{\left[i^{\prime} n\right]}}\right)\left(\sum_{m=1}^{2 M} \sigma_{m} x_{m} \hat{\mathbf{h}}_{l}^{H} \mathbf{h}_{m}\right)\right\}}_{\text {part } 2} .
\end{aligned}
$$

Proof:

$$
\begin{aligned}
& \mathbb{E}\left[\left|\sum_{m=1, m \neq i}^{2 M} \sigma_{m} x_{m} \hat{\mathbf{h}}_{i}^{H} \mathbf{h}_{m}\right|^{K}\right] \\
& =\mathbb{E}\left[\left|\sum_{m=1, m \neq i}^{2 M}\left(\sigma_{m} x_{m} \sum_{p=1}^{N} \hat{h}_{p i}^{*} h_{p m}\right)\right|^{K}\right] \\
& \stackrel{(a)}{=} \sum_{1 \leq i_{1}, . ., i_{k} \leq(2 M-1) N} \mathbb{E}\left[X_{i_{1}} X_{i_{2}}^{*} \cdots X_{i_{K-1}} X_{i_{K}}^{*}\right] \\
& \stackrel{(b)}{\approx} O\left[(2 M-1)^{\frac{K}{2}} N^{\frac{K}{2}}\right] \mathbb{E}[\underbrace{\left[\left.\sigma_{m} x_{m} \hat{h}_{p i}^{*} h_{p m}\right|^{2} \cdots\left|\sigma_{n} x_{n} \hat{h}_{q i}^{*} h_{q n}\right|^{2}\right.}_{\frac{K}{2} \text { terms }}] \\
& \stackrel{(c)}{<} O\left[(2 M-1)^{\frac{K}{2}} N^{\frac{K}{2}}\right] \tilde{k}_{2} .
\end{aligned}
$$

Here the $X_{i}$ 's in $(a)$ take values of the type $\sigma_{m} x_{m} \hat{h}_{p i}^{*} h_{p m}$. Since $h_{p i}$ 's and $\left[E_{u}\right]_{p i}$ are iid $\mathcal{C N}(0,1)$ and $\mathcal{C N}\left(0, \eta_{u}^{2}\right)$ respectively, and $\hat{h}_{p i}=\sigma_{i} h_{p i}+\left[E_{u}\right]_{p i}$, each $\sigma_{m} x_{m} \hat{h}_{p i}^{*} h_{p m}$ term must occur exactly twice to contribute to the $O\left[(2 M-1)^{\frac{K}{2}} N^{\frac{K}{2}}\right]$ number of dominant terms in the approximation in step $(b)$. The approximation is tight for large $N$ and $2 M$ [19]. The finite constant $\tilde{k}_{2}$ bounds the expectation term in the particular term, which precedes the inequality $(b)$. Inequality in $(c)$ is true, since all moments of Gaussian random variables $h_{p i}$ 's and $\hat{h}_{p i}=\sigma_{i} h_{p i}+\left[E_{u}\right]_{p i}$ are finite.

Lemma A.4:

$$
\begin{aligned}
& \mathbb{E}[\underbrace{\left.\left.\sum_{m=1}^{2 M} \sigma_{m} x_{m} \hat{\mathbf{h}}_{l}^{H} \mathbf{h}_{m}\right|^{2} \cdots\left|\sum_{m=1}^{2 M} \sigma_{m} x_{m} \hat{\mathbf{h}}_{l^{\prime}}^{H} \mathbf{h}_{m}\right|^{2}\right]}_{\frac{K}{2} \text { terms }} \\
& <\left(O\left[N^{K}\right]+O\left[(2 M-1)^{\frac{K}{2}}(N)^{\frac{K}{2}}\right]\right) \tilde{k}_{2} .
\end{aligned}
$$

Proof: Similar to the bounds derived in Lemma A.3.

Combining equation (A.3) with Lemma A.3 and Lemma A.4 we have

$$
\begin{aligned}
& \frac{\mathbb{E}\left[\left|\tilde{R}_{I_{i^{\prime}}}\right|^{K}\right]}{c^{K} \sigma_{i^{\prime}}^{K}} \\
& <O\left[(2 M-1)^{\frac{K}{2}} N^{\frac{3 K}{2}}\right] \tilde{k}+O\left[(2 M-1)^{K}(N)^{K}\right] \tilde{\tilde{k}},
\end{aligned}
$$

where $\tilde{k}=2 \tilde{k}_{1} \tilde{k}_{2}$ and $\tilde{\tilde{k}}=\tilde{k}_{1} \tilde{k}_{2}$ are constants.

\section{APPENDIX B \\ Proof OF $\mathbb{E}\left[e^{j \theta_{n l}}\right]=0$}

Given that the channel estimates are corrupted by AWGN, $e^{j \theta_{n i^{\prime}}}$ is a zero-mean random variable i.e., $\mathbb{E}\left[e^{j \theta_{n i^{\prime}}}\right]=0$, $\forall n, i^{\prime}$.

Proof: As mentioned in Section II, we assume that the relay has a quantized estimate of the DL channel $\mathbf{D}^{\frac{1}{2}} \mathbf{G}$. The DL channel estimated by user $i^{\prime}$ is $\hat{g}_{i^{\prime} n}$, which is given as $\hat{g}_{i^{\prime} n}=\sigma_{i^{\prime}} g_{i^{\prime} n}+\tilde{e}_{i^{\prime} n}=\left|\hat{g}_{i^{\prime} n}\right| e^{j \hat{\alpha}_{i^{\prime} n}}$. Here $\sigma_{i^{\prime}} g_{i^{\prime} n}$ is the true value and $\tilde{e}_{i^{\prime} n}$ is the $\left(i^{\prime}, n\right)$ th entry of $\mathbf{E}_{d}$ distributed iid $\mathcal{C N}\left(0, \eta_{d}^{2}\right)$ [13]. The pdf of $\hat{\alpha}_{i^{\prime} n}$ given $\alpha_{i^{\prime} n}, \sigma_{i^{\prime}}\left|g_{i^{\prime} n}\right|$ as derived in [31] is

$$
\lambda\left(\hat{\alpha}_{i^{\prime} n} / \alpha_{i^{\prime} n}, \sigma_{i^{\prime}}\left|g_{i^{\prime} n}\right|\right)=\int_{0}^{\infty} t e^{\frac{2 r \sigma_{i^{\prime}}\left|g_{i^{\prime} n}\right| \cos \left(\hat{(}_{i^{\prime} n}-\alpha_{i^{\prime} n}\right)}{\eta_{d}^{2}}} d r
$$

where $t=\frac{r}{\pi \eta_{d}^{2}} e^{\frac{-r^{2}-\sigma_{i^{\prime}}^{2}\left|g_{i^{\prime} n}\right|^{2}}{\eta_{d}^{2}}}$. Now, we have:

$\mathbb{E}\left[e^{j \theta_{n i^{\prime}}}\right]=\mathbb{E}_{\left|g_{i^{\prime} n}\right|} \mathbb{E}_{\alpha_{i^{\prime} n}} \mathbb{E}_{\hat{\alpha}_{i^{\prime} n}}\left[e^{j \theta_{n i^{\prime}}}\right]$

$=\mathbb{E}_{\mid g_{i^{\prime} n} n} \mid \mathbb{E}_{\alpha_{i^{\prime} n}} \int_{-\pi}^{\pi} e^{j \theta_{n i^{\prime}}} \lambda\left(\hat{\alpha}_{i^{\prime} n} / \alpha_{i^{\prime} n}, \sigma_{i^{\prime}}\left|g_{i^{\prime} n}\right|\right) d \hat{\alpha}_{i^{\prime} n}$

$=\mathbb{E}_{\left|g_{i^{\prime} n}\right|} \mid \frac{1}{2 \pi} \int_{-\pi}^{\pi}\left(\int_{-\pi}^{\pi} e^{j \theta_{n i^{\prime}}} \lambda\left(\hat{\alpha}_{i^{\prime} n} / \alpha_{i^{\prime} n}, \sigma_{i^{\prime}}\left|g_{i^{\prime} n}\right|\right) d \hat{\alpha}_{i^{\prime} n}\right) d \alpha_{i^{\prime} n}$

$=\mathbb{E}_{\left|g_{i^{\prime} n}\right|}[I]$. (let)

Note from (14) that whenever,

$\hat{\alpha}_{i^{\prime} n} \in S_{k}=\left[\pi-\left(k-\frac{1}{2}\right) \frac{2 \pi}{2^{B}}, \pi-\left(k-\frac{3}{2}\right) \frac{2 \pi}{2^{B}}\right)$, we have $\theta_{n i^{\prime}}\left(\hat{\alpha}_{i^{\prime} n}\right)=-\pi+(k-1) \frac{2 \pi}{2^{B}}$.

This implies that

$$
\hat{\alpha}_{i^{\prime} n}+\pi \in\left[2 \pi-\left(k-\frac{1}{2}\right) \frac{2 \pi}{2^{B}}, 2 \pi-\left(k-\frac{3}{2}\right) \frac{2 \pi}{2^{B}}\right)
$$

forcing

$$
\theta_{n i^{\prime}}\left(\hat{\alpha}_{i^{\prime} n}+\pi\right)=-2 \pi+(k-1) \frac{2 \pi}{2^{B}}=-\pi+\theta_{n i^{\prime}}\left(\hat{\alpha}_{i^{\prime} n}\right) .
$$


Hence for every $\left(\hat{\alpha}_{i^{\prime} n}, \alpha_{i^{\prime} n}, \sigma_{i^{\prime}}\left|g_{i^{\prime} n}\right|\right)$ tuple, by (B.1),

$$
\begin{array}{ll}
e^{j \theta_{n i^{\prime}}\left(\hat{\alpha}_{i^{\prime} n}\right)} \lambda\left(\hat{\alpha}_{i^{\prime} n} / \alpha_{i^{\prime} n}, \sigma_{i^{\prime}}\left|g_{i^{\prime} n}\right|\right) & \text { of } \\
=-e^{j \theta_{n i^{\prime}}\left(\hat{\alpha}_{i^{\prime} n}+\pi\right)} \lambda\left(\hat{\alpha}_{i^{\prime} n}+\pi / \alpha_{i^{\prime} n}+\pi, \sigma_{i^{\prime}}\left|g_{i^{\prime} n}\right|\right) & \mathbb{E}\left[\mid h_{h}\right. \\
\Rightarrow \int_{-\pi}^{\pi} \int_{-\pi}^{\pi} e^{j \theta_{n i^{\prime}}\left(\hat{\alpha}_{i^{\prime} n}\right)} \lambda\left(\hat{\alpha}_{i^{\prime} n} / \alpha_{i^{\prime} n}, \sigma_{i^{\prime}}\left|g_{i^{\prime} n}\right|\right) d \hat{\alpha}_{i^{\prime} n} d \alpha_{i^{\prime} n} & \text { shown } \\
= & -\int_{-\pi}^{\pi} \int_{-\pi}^{\pi} e^{j \theta_{n i^{\prime}}\left(\hat{\alpha}_{i^{\prime} n}+\pi\right)} \lambda\left(\hat{\alpha}_{i^{\prime} n}+\pi / \alpha_{i^{\prime} n}+\pi, \sigma_{i^{\prime}}\left|g_{i^{\prime} n}\right|\right) d \hat{\alpha}_{i^{\prime} n} d \alpha_{i^{\prime} n} \\
\Rightarrow I=-I \Rightarrow I=0 .
\end{array}
$$

Hence the result follows by substituting (B.3) into (B.2). For complex zero-mean non-Gaussian distributions, the result holds true if the pdf $f_{\alpha_{i^{\prime} n}}($.$) of \alpha_{i^{\prime} n}$ satisfies $f_{\alpha_{i^{\prime} n}}(x)=$ $f_{\alpha_{i^{\prime} n}}(x+\pi)$ and is independent of $\left|g_{i^{\prime} n}\right|$.

\section{APPENDIX C$$
\text { BOUND ON } \mathbb{E}\left[\left|\tilde{N}_{I_{i^{\prime}}}\right|^{K}\right]
$$

Claim: For even $K$ and for large $N, 2 M$, the relay noise term $\tilde{N}_{I_{i^{\prime}}}$ defined in (22) is bounded by: $\frac{\mathbb{E}\left[\left|\tilde{N}_{I_{i^{\prime}}}\right|^{K}\right]}{c^{K} \sigma_{i^{\prime}}^{K}}<$ $O\left[N^{\frac{3 K}{2}}\right] \tilde{k}+O\left[(2 M)^{\frac{K}{2}} N^{K}\right] \tilde{k}$.

Proof: Expanding (22) using $K$ moment expansion formula [19], we have, $\frac{\mathbb{E}\left[\left|\tilde{N}_{I_{i^{\prime}}}\right|^{K}\right]}{c^{K} \sigma_{i^{\prime}}^{K}}$

$$
\begin{aligned}
& \stackrel{(a)}{=} O\left[N^{K}\right] \mathbb{E}[\underbrace{\left|g g_{i^{\prime} p}\right| e^{j \delta_{p i^{\prime}}^{\left[i^{\prime} p\right]}} \tilde{n}_{i} \cdots\left|g_{i^{\prime} p^{\prime}}\right| e^{-j \delta_{p^{\prime} i^{\prime}}^{\left[i^{\prime} p^{\prime}\right]}} \tilde{n}_{i}}_{K \text { terms }}] \\
& +O\left[(2 M)^{\frac{K}{2}} N^{\frac{K}{2}}\right] \mathbb{E}[\underbrace{\left|g_{i^{\prime} p^{\prime}}\right|^{2}\left|\tilde{n}_{l}\right|^{2} \cdots\left|g_{i^{\prime} p^{\prime \prime}}\right|^{2}\left|\tilde{n}_{l^{\prime}}\right|^{2}}_{\frac{K}{2} \text { terms }}] \\
& \stackrel{(b)}{=} O\left[N^{K}\right] \mathbb{E}\left[\left|g_{i^{\prime} p}\right| e^{j \delta_{p i^{\prime}}^{\left[i^{\prime} p\right]}} \cdots\left|g_{i^{\prime} p^{\prime}}\right| e^{-j \delta_{p^{\prime} i^{\prime}}^{\left[i^{\prime} p^{\prime}\right]}}\right] \mathbb{E}\left[\left|\tilde{n}_{i}\right|^{K}\right] \\
& +O\left[(2 M)^{\frac{K}{2}} N^{\frac{K}{2}}\right] \mathbb{E}\left[\left|g_{i^{\prime} p^{\prime}}\right|^{2} \cdots\left|g_{i^{\prime} p^{\prime \prime}}\right|^{2}\right] \mathbb{E}\left[\left|\tilde{n}_{l}\right|^{2} \cdots\left|\tilde{n}_{l^{\prime}}\right|^{2}\right] \\
& \stackrel{(c)}{<} O\left[N^{K}\right] \mathbb{E}\left[\left|\tilde{n}_{i}\right|^{K}\right] \tilde{k}_{3}+O\left[(2 M)^{\frac{K}{2}} N^{\frac{K}{2}}\right] \mathbb{E}\left[\left|\tilde{n}_{l}\right|^{2} \cdots\left|\tilde{n}_{l^{\prime}}\right|^{2}\right] \tilde{k}_{3} .
\end{aligned}
$$

Note that $\left|g_{i^{\prime} p}\right| e^{j \delta_{p i^{\prime}}^{\left[i^{\prime} p\right]}}$ are non-zero mean random variables. They contribute to the dominant term in $O\left(N^{K}\right)$ ways in the expansion of the sum in step $(a) .\left|g_{i^{\prime} p}\right| e^{j \delta_{p l^{\prime}}^{\left[i^{\prime} p\right]}}$ are zeromean random variables, where each such independent term must occur exactly twice along with its conjugate in the sum in step $(a)$ to contribute to the dominant term. The number of such terms is $O\left[(2 M)^{\frac{K}{2}} N^{\frac{K}{2}}\right]$. Equality in (b) is because that the entries of $\widehat{\mathbf{H}}, \mathbf{G}$ and $\mathbf{n}_{r}$ are independent of each other and $\tilde{\mathbf{n}}_{l}=\hat{\mathbf{h}}_{l} n_{r}$. Constant $\tilde{k}_{3}$ occurs in inequality in (c) true because

$$
\mathbb{E}\left[\left|g_{i^{\prime} p}\right| e^{j \delta_{p i^{\prime}}^{\left[i^{\prime} p\right]}} \cdots\left|g_{i^{\prime} p^{\prime}}\right| e^{-j \delta_{p^{\prime} i}^{\left[i^{\prime} p^{\prime}\right]}}\right] \leq \sqrt{\mathbb{E}\left[\left|g_{i^{\prime} p}\right|^{2} \cdots\left|g_{i^{\prime} p^{\prime}}\right|^{2}\right]},
$$

and the fact that all moments of Gaussian random variables can be bounded. Next, we have

$$
\begin{aligned}
& \mathbb{E}\left[\left|\tilde{n}_{i}\right|^{K}\right]=\mathbb{E}\left[\left|\sum_{k=1}^{N} \hat{h}_{k i}^{*} n_{r_{k}}\right|^{K}\right] \\
& =O\left(N^{\frac{K}{2}}\right) \mathbb{E}\left[\left|\hat{h}_{k i}\right|^{2}\left|n_{r_{k}}\right|^{2} \cdots\left|\hat{h}_{k^{\prime} i}\right|^{2}\left|n_{r_{k^{\prime}}}\right|^{2}\right] \\
& <O\left(N^{\frac{K}{2}}\right) \tilde{k}_{4} .
\end{aligned}
$$

Equation (C.2) follows from the fact that all finite moments of Gaussian random variables $\hat{h}_{k i}, n_{r_{k}}$ can be bounded i.e., $\mathbb{E}\left[\left|\hat{h}_{k i}\right|^{2}\left|n_{r_{k}}\right|^{2} \cdots\left|\hat{h}_{k^{\prime} i}\right|^{2}\left|n_{r_{k^{\prime}}}\right|^{2}\right]<\tilde{k}_{3}$. Likewise, it can be shown that

$$
\mathbb{E}\left[\left|\tilde{n}_{i}\right|^{2} \cdots\left|\tilde{n}_{l}\right|^{2}\right]<O\left[N^{\frac{K}{2}}\right] \tilde{k}_{4}
$$

From (C.1), (C.2) and (C.3), we have

$$
\frac{\mathbb{E}\left[\left|\tilde{N}_{I_{i^{\prime}}}\right|^{K}\right]}{c^{K} \sigma_{i^{\prime}}^{K}}<O\left[N^{\frac{3 K}{2}}\right] \tilde{k}+O\left[(2 M)^{\frac{K}{2}} N^{K}\right] \tilde{k}
$$

where $\tilde{k}=\tilde{k}_{3} \tilde{k}_{4}$.

\section{APPENDIX D}

PROOF OF EQUATION (41)

\section{Claim:}

$$
\begin{aligned}
& \mathbb{E}\left[\lim _{N \rightarrow \infty} \frac{\sum_{i^{\prime}=1}^{N^{\alpha}} \log \left(1+\frac{p_{i^{\prime}}^{2}}{\left|\tilde{R}_{I_{i^{\prime}}}\right|^{2}+\left|\tilde{N}_{I_{i^{\prime}}}\right|^{2}+N_{0}}\right)}{N^{\alpha}}\right] \\
& =\lim _{N \rightarrow \infty} \frac{\sum_{i^{\prime}=1}^{N^{\alpha}} \log \left(1+\frac{E_{r} \zeta_{i, i^{\prime}}^{2}}{N_{0}}\right)}{N^{\alpha}} .
\end{aligned}
$$

Proof: We know from (21) that the interference power obeys $\left|\tilde{R}_{I_{i^{\prime}}}\right|^{2} \stackrel{a . s .}{\rightarrow} 0$ as $N \rightarrow \infty$. Hence for $\epsilon>0, \exists \widehat{N_{1}}(\epsilon)$ such that $\forall N \geq \widehat{N_{1}}(\epsilon)$ the event $\left\{\left|\tilde{R}_{I_{i^{\prime}}}\right|^{2}>\epsilon\right\}$ almost surely never occurs. Furthermore, Remark 7 tells us that the $\widehat{N_{1}}(\epsilon)$ threshold is the same for all the $N^{\alpha}$ users.

Likewise from (23) and (25), for $\epsilon>0, \exists \widehat{N_{2}}(\epsilon)$ such that $\forall N \geq \widehat{N_{2}}(\epsilon)$ the event $\left\{\left|\tilde{N}_{I_{i^{\prime}}}\right|^{2}>\epsilon\right\}$ almost surely never occurs. This $\widehat{N_{2}}(\epsilon)$ threshold is the same for all the $N^{\alpha}$ users by Remark 7.

Similarly the received signal power $p_{i^{\prime}}^{2}$ converges almost surely to $\left(\sigma_{i}^{2} \sigma_{i^{\prime}} \mathbb{E}\left[\left|g_{i^{\prime} n}\right| e^{j \delta_{n i^{\prime}}^{\left[i^{\prime} n\right]}}\right]\right)^{2}\left(\frac{E_{r}}{\lim _{N \rightarrow \infty} \frac{\sum_{i=1}^{N^{\alpha}} \sigma_{i}^{4}}{N^{\alpha}}}\right)=$ $E_{r} \zeta_{i, i^{\prime}}^{2}$. Hence for $\epsilon>0, \exists \widehat{N_{3}}(\epsilon)$ such that $\forall N \geq \widehat{N_{3}}(\epsilon)$ the event $\left\{\left|p_{i^{\prime}}^{2}-E_{r} \zeta_{i, i^{\prime}}^{2}\right|>\epsilon\right\}$ almost surely never occurs. Also the convergence is uniform for all users.

From the above discussions we conclude that for $\epsilon>$ $0, \exists \widehat{N_{4}}(\epsilon)=\max \left\{\widehat{N_{1}}(\epsilon), \widehat{N_{2}}(\epsilon), \widehat{N_{3}}(\epsilon)\right\}$ such that $\forall N \geq$ $N_{4}(\epsilon)$ the event $\left\{\left|\frac{p_{i^{\prime}}^{2}}{\left|\tilde{R}_{I_{i^{\prime}}}\right|^{2}+\left|\tilde{N}_{\left.I_{i^{\prime}}\right|^{2}}\right|^{2}+N_{0}}-\frac{E_{r} \zeta_{i, i^{\prime}}^{2}}{N_{0}}\right|>\epsilon\right\}$ almost surely never occurs.

Next, since $\log (1+x)$ is continuous in $x, \exists \widehat{N}(\epsilon)$ such that $\forall N \geq \widehat{N}(\epsilon)$ such that the event

$$
\left\{\left|\log \left(1+\frac{p_{i^{\prime}}^{2}}{\left|\tilde{R}_{I_{i^{\prime}}}\right|^{2}+\left|\tilde{N}_{I_{i^{\prime}}}\right|^{2}+N_{0}}\right)-\log \left(1+\frac{E_{r} \zeta_{i, i^{\prime}}^{2}}{N_{0}}\right)\right|>\epsilon\right\}
$$

almost surely never occurs. 
Now $\forall N \geq \widehat{N}(\epsilon)$,

$$
\begin{aligned}
& \frac{\left|\sum_{i^{\prime}=1}^{N^{\alpha}} \log \left(1+\frac{p_{i^{\prime}}^{2}}{\left|\tilde{R}_{I_{i^{\prime}}}\right|^{2}+\left|\tilde{N}_{I^{\prime}}\right|^{2}+N_{0}}\right)-\log \left(1+\frac{E_{r} \zeta_{i, i^{\prime}}^{2}}{N_{0}}\right)\right|}{N^{\alpha}} \\
& \leq \frac{\sum_{i^{\prime}=1}^{N^{\alpha}}\left|\log \left(1+\frac{p_{i^{\prime}}^{2}}{\left|\tilde{R}_{I_{i^{\prime}}}\right|^{2}+\left|\tilde{N}_{i^{\prime}}\right|^{2}+N_{0}}\right)-\log \left(1+\frac{E_{r} \zeta_{i, i^{\prime}}^{2}}{N_{0}}\right)\right|}{N^{\alpha}}
\end{aligned}
$$

(a)

$\leq \epsilon$.

Step $(a)$ is true because (D.1) holds for all users uniformly. The result, thus holds true.

\section{REFERENCES}

[1] A. K. Dutta, K. V. S. Hari, and L. Hanzo, "Linear transceiver design for an amplify-and-forward relay based on the MBER criterion," IEEE Trans. Commun., vol. 62, no. 11, pp. 3765-3777, Nov. 2014.

[2] P. Das and N. B. Mehta, "Direct link-aware optimal relay selection and a low feedback variant for underlay CR," IEEE Trans. Commun., vol. 63, no. 6, pp. 2044-2055, Jun. 2015

[3] B. Rankov and A. Wittneben, "Spectral efficient protocols for halfduplex fading relay channels," IEEE J. Sel. Areas Commun., vol. 25, no. 2, pp. 379-389, Feb. 2007.

[4] R. Budhiraja and B. Ramamurthi, "Joint transceiver design for QoSconstrained MIMO two-way non-regenerative relaying using geometric programming," IEEE Trans. Wireless Commun., vol. 15, no. 5, pp. 34533465, May 2016.

[5] J. Joung and A. H. Sayed, "Multiuser two-way amplify-and-forward relay processing and power control methods for beamforming systems," IEEE Trans. Signal Process., vol. 58, no. 3, pp. 1833-1846, Mar. 2010.

[6] K. X. Nguyen, Y. Rong, and S. Nordholm, "Simplified MMSE precoding design in interference two-way MIMO relay systems," IEEE Signal Processing Letters, vol. 23, no. 2, pp. 262-266, Feb. 2016.

[7] D. G. et.al, "The multiway relay channel," IEEE Trans. Inf. Theory, vol. 59, no. 1, pp. 51-63, Jan. 2013.

[8] Z. Fang, X. Wang, and X. Yuan, "Beamforming design for multiuser two-way relaying: A unified approach via max-min SINR," IEEE Trans. Signal Process., vol. 61, no. 23, pp. 5841-5852, Dec. 2013.

[9] X. Yuan, "MIMO multiway relaying with clustered full data exchange: Signal space alignment and degrees of freedom," IEEE Trans. Wireless Commun., vol. 12, no. 12, pp. 6795-6808, Dec. 2014.

[10] F. Rusek, D. Persson, B. K. Lau, E. G. Larsson, T. L. Marzetta, O. Edfors, and F. Tufvesson, "Scaling up MIMO: Opportunities and challenges with very large arrays," IEEE Signal Process. Mag., vol. 30, no. 1, pp. 40-60, Jan. 2013.

[11] E. Bjrnson, J. Hoydis, and L. Sanguinetti, "Massive MIMO networks: Spectral, energy, and hardware efficiency," Foundations and Trends in Signal Processing, vol. 11, no. 3-4, pp. 154-655, Nov. 2017.

[12] H. Cui, L. Song, and B. Jiao, "Multi-pair two-way amplify-and-forward relaying with very large number of relay antennas," IEEE Trans. Wireless Commun., vol. 13, no. 5, pp. 2636-2645, May 2014.

[13] Y. Dai and X. Dong, "Power allocation for multi-pair massive MIMO two-way AF relaying with linear processing," IEEE Trans. Wireless Commun., vol. 15, no. 9, pp. 5932-5946, Sep. 2016.

[14] J. Liu, W. Xu, S. Jin, and X. Dong, "RF-chain constrained multi-pair massive MIMO relaying using hybrid precoding and detection," in 2016 IEEE Wireless Communications and Networking Conference, Apr. 2016, pp. 1-6.

[15] C. Kong, C. Zhong, M. Matthaiou, E. Bjornson, and Z. Zhang, "Multipair two-way AF relaying systems with massive arrays and imperfect CSI," in 2016 IEEE International Conference on Acoustics, Speech and Signal Processing (ICASSP), Mar. 2016, pp. 3651-3655.

[16] S. Jin, X. Liang, K. K. Wong, X. Gao, and Q. Zhu, "Ergodic rate analysis for multipair massive MIMO two-way relay networks," IEEE Trans. Wireless Commun., vol. 14, no. 3, pp. 1480-1491, Mar. 2015.

[17] M. M. Naghsh, M. Soltanalian, P. Stoica, M. Masjedi, and B. Ottersten, "Efficient sum-rate maximization for medium-scale MIMO AF-relay networks," IEEE Trans. Wireless Commun., vol. 15, no. 9, pp. 64006411, Sep. 2016.

[18] E. Sharma, R. Budhiraja, K. Vasudevan, and L. Hanzo, "Full-duplex massive MIMO multi-pair two-way AF relaying: Energy efficiency optimization," IEEE Trans. Commun., accepted, to appear in 2018.
[19] T. Tao, Topics in Random Matrix Theory, ser. Graduate studies in mathematics. American Mathematical Soc.

[20] D. Williams, Probability with Martingales. Cambridge university press, 1991.

[21] P. Chandhar, D. Danev, and E. G. Larsson, "Massive mimo as enabler for communications with drone swarms," in 2016 International Conference on Unmanned Aircraft Systems (ICUAS), June 2016, pp. 347-354.

[22] A. S. G. Andrae and T. Edler, "On global electricity usage of communication technology: Trends to 2030," Challenges, vol. 6, no. 1, pp. 117-157, 2015.

[23] B. A. et.al, "Challenges toward wireless communications for high-speed railway," IEEE Transactions on Intelligent Transportation Systems, vol. 15, no. 5, pp. 2143-2158, Oct 2014.

[24] M. Abuthinien, S. Chen, and L. Hanzo, "Semi-blind joint maximum likelihood channel estimation and data detection for MIMO systems," IEEE Signal Processing Letters, vol. 15, pp. 202-205, 2008.

[25] C. Cozzo and B. L. Hughes, "Joint channel estimation and data detection in space-time communications," IEEE Trans. Commun., vol. 51, no. 8, pp. 1266-1270, Aug. 2003.

[26] M. Vu and A. Paulraj, "MIMO wireless linear precoding," IEEE Signal Process. Mag., vol. 24, no. 5, Sep. 2007.

[27] S. Sesia, I. Toufik, and M. Baker, LTE-The UMTS Long Term Evolution. West Sussex, UK: Wiley, 2011.

[28] B. Dutta, "Full-diversity achieving precoding.... partial CSIT," IEEE Trans. Commun., vol. 61, no. 3, Mar. 2013.

[29] D. Tse and P. Viswanath, Fundamentals of Wireless Communication. Cambridge university press, 2005.

[30] T. S. Rappaport, Wireless Communications: Principles and Practice, 1st ed. Piscataway, NJ, USA: IEEE Press, 1996.

[31] P. Beckmann, "Statistical distribution of the amplitude and phase of a multiply scattered field," Journal of Research of the National Bureau of Standards, vol. 66D, no. 3, pp. 231-240, Jun. 1962.

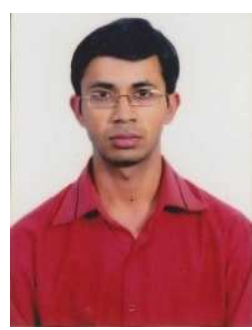

Biswajit Dutta received the B.E. degree in Electronics and Telecommunication Engineering from Jadavpur University, Kolkata, India in 2007. He received the M.E. degree in Telecommunication Engineering from Indian Institute Of Science, Bangalore, India in 2009. He is currently pursuing his $\mathrm{Ph} . \mathrm{D}$. from Indian Institute of Technology Madras, India. His research interests include wireless communications and social networks. He has worked in indoor position verification algorithms in Pipal Tech Ventures Pvt. Ltd. and in plant load scheduling optimization software in Bharat Heavy Electricals Ltd from 2009 to 2015.

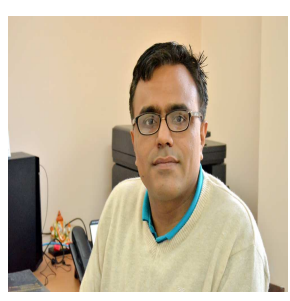

Rohit Budhiraja received his M.S. in Electrical Engineering from IIT Madras in 2004. From January 2004 to July 2011, he worked for two startups where he designed both hardware and software algorithms, from scratch, for physical layer processing of WiMAX- and LTE-based cellular systems. He worked on his Ph.D. from August 2011 to July 2015 at IIT Madras. He is currently an Assistant Professor and a Visvesvaraya Fellow at IIT Kanpur. $\mathrm{He}$ is also leading an effort to design a $5 \mathrm{G}$ research testbed at IIT Kanpur. His current research interests include design and analysis of for 5G massive MIMO, full-duplex, and NOMA systems, robust transceiver design for wireless relaying, machine learning methods for mm-wave/cloud radio systems, and spatial modulation system design. His paper was shortlisted as one of the finalists for the Best Student Paper Awards at IEEE International Conference on Signal Processing and Communications (SPCOM), Bangalore, India, 2014. He also received IIT Madras research award for the quality and quantity of research work done in the Ph.D., Early Career Research Award, and teaching excellence certificate at IIT Kanpur. 
Ravinder D. Koilpillai received the B.Tech degree in Electrical Engineering from the Indian Institute of Technology Madras and the M.S. and Ph.D. degrees in Electrical Engineering from the California Institute of Technology, Pasadena, CA.

In June 2002, David joined the EE faculty of IIT Madras. He is currently the Qualcomm Institute Chair Professor in EE and Dean (Planning). During the period April 2008 December 2009, he served as the Co-Chair of the IITM special Task Force for setting up the new IIT at Hyderabad. David also served as Head, Central Electronics Centre of IITM during 20010-11. Davids technical areas of expertise include cellular and broadband wireless systems, and DSP techniques for wireless communications. He is the Faculty Coordinator of the IITMSAT Student Satellite initiative. During January July 2007, David was on sabbatical from IITM and served as the Chief Scientist, Centre of Excellence in Wireless Technology (CEWiT), a publicprivate R\&D initiative of the Govt. of India, and was responsible for launching the national project Broadband Wireless Consortium of India (BWCI). Prior to joining IITM, David was at General Electric Corporate R\&D for four years and Ericsson USA for eight years, where he held different technical and managerial positions. In 2000, he became the Director of the Ericssons Advanced Technologies and Research Department at RTP, North Carolina, developing GPRS/EDGE handset technology.

Davids technical contributions at GE and Ericsson have resulted in 32 US patents, 10 Canadian Patents and 19 WIPO/European patents. In 1999 David received the Ericsson Inventor of the Year award, the highest technical recognition within Ericsson. In Nov 2003 David was elected Fellow of the Indian National Academy of Engineering. In 2014, David received the Srimathi Marti Annapurna Gurunath Award for Excellence in Teaching (Best Teacher Award of IIT Madras)

Davids current technical activities are in the areas of Cellular evolution - 4G and 5G, Smart grid Communications, DSP for High Speed Coherent optical communications

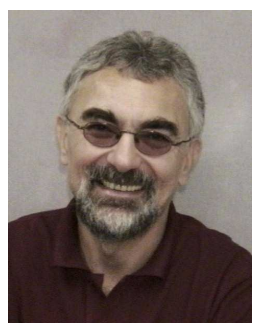

Lajos Hanzo (http://www-mobile.ecs.soton.ac.uk) FREng, F'04, FIET, Fellow of EURASIP, received his 5-year degree in electronics in 1976 and his doctorate in 1983 from the Technical University of Budapest. In 2009 he was awarded an honorary doctorate by the Technical University of Budapest and in 2015 by the University of Edinburgh. In 2016 he was admitted to the Hungarian Academy of Science. During his 40-year career in telecommunications he has held various research and academic posts in Hungary, Germany and the UK. Since 1986 he has been with the School of Electronics and Computer Science, University of Southampton, UK, where he holds the chair in telecommunications. He has successfully supervised $112 \mathrm{PhD}$ students, co-authored 18 John Wiley/IEEE Press books on mobile radio communications totalling in excess of 10000 pages, published 1790 research contributions at IEEE Xplore, acted both as TPC and General Chair of IEEE conferences, presented keynote lectures and has been awarded a number of distinctions. Currently he is directing an academic research team, working on a range of research projects in the field of wireless multimedia communications sponsored by industry, the Engineering and Physical Sciences Research Council (EPSRC) UK, the European Research Council's Advanced Fellow Grant and the Royal Society's Wolfson Research Merit Award. He is an enthusiastic supporter of industrial and academic liaison and he offers a range of industrial courses. He is also a Governor of the IEEE ComSoc and VTS. During 2008 - 2012 he was the Editor-in-Chief of the IEEE Press and a Chaired Professor also at Tsinghua University, Beijing. For further information on research in progress and associated publications please refer to http://www-mobile.ecs.soton.ac.uk 